\title{
Understanding the relevance of national culture in international business research: A quantitative analysis
}

\author{
López-Duarte, Cristina* \\ University of Oviedo, Department of Business \\ clduarte@uniovi.es, +34985182179 \\ Fc. Commerce, Tourism and Social Sciences \\ Av. Luís Moya, 33203 Gijón, Asturias, Spain \\ Vidal-Suárez, Marta M. \\ University of Oviedo, Department of Business \\ mmvidal@uniovi.es, +34985182302 \\ Fc Commerce, Tourism and Social Sciences \\ Av. Luís Moya, 33203 Gijón, Asturias, Spain \\ González-Díaz, Belén. \\ University of Oviedo, Department of Accountancy \\ bgonzal@uniovi.es, +34985182176 \\ Fc Commerce, Tourism and Social Sciences \\ Av. Luís Moya, 33203 Gijón, Asturias, Spain
Nuno Rosa Reis
Instituto Politécnico de Leiria, Escola Superior de Tecnologia e Gestão, nuno.m.reis@ipleiria.pt, +351244820300
Morro do Lena - Alto Vieiro
2411-901 Leiria, Portugal

* Corresponding author

The final version of the paper is available at https://doi.org/10.1007/s11192-016-2044-9 


\title{
Understanding the relevance of national culture in International Business research: A quantitative analysis
}

\begin{abstract}
This review is a comprehensive quantitative analysis of the International Business literature whose focus is on national culture. The analysis relies on a broad range of bibliometric techniques as productivity rankings, citation analysis (individual and cumulative), study of collaborative research patterns, and analysis of the knowledge base. It provides insights on (I) faculty and institutional research productivity and performance; (II) articles, institutions, and scholars' influence in the contents of the field and its research agenda; and (III) national and international collaborative research trends. The study also explores the body of literature that has exerted the greatest impact on the researched set of selected articles.
\end{abstract}

Key words: Quantitative analysis, national culture, international business, bibliometric, citation analysis 


\section{INTRODUCTION}

The role played by national culture (NC) and cultural differences and distances (CD) in a wide range of International Business (IB) decisions has been researched extensively in existing literature. As a consequence, some recent literature reviews within the IB field have focused their attention on this issue. Some of these reviews center their attention on a specific topic as, for instance, the impact of cultural differences on the mode used to enter the target markets, the performance of the internationalization processes, or the way of managing shared ventures - e.g.: Harzing (2003); Morschett et al. (2010), Reus and Rottig (2009); Shenkar (2001); Tihanyi et al. (2005). Others show a wider perspective, as the recent review published in the International Journal of Management Reviews by López-Duarte, et al. (2015) — LVG from here- aimed at mapping the entire field (e.g.: international growth decisions, choice of target countries, alternative entry modes features and performance, human resource management, organizational design, knowledge transfer).

LVG (2015) is a study developed from a subjective approach based on a qualitative analysis of 265 selected articles published in 26 leading IB and Management journals from 2000 to $2012^{1}$. As a qualitative study, it relies on a content analysis of the articles selected by the research team. Therefore, it can be expected that the final output reflects the subjective views and interpretations of its authors (Ramos-Rodríguez and Ruíz-Navarro, 2004). In other words, when a literature review is developed from a qualitative approach, potential personal biases arise as the researchers

\footnotetext{
${ }^{1}$ See LVG (2015) for an exhaustive description of the process and criteria followed to select both the journals and the articles included in the researched dataset. In short, the list of selected journals includes the International Business Review (IBR), International Marketing Review (IMR), Journal of International Business Studies (JIBS), Journal of International Management (JIMn), Journal of International Marketing (JIMr), Journal of World Business $(J W B)$, and Management International Review (MIR), as well as the 20-top academic management journals initially classified by Gómez-Mejía and Balkin (1992) and later reviewed by Pisani (2011) and Werner (2002). The dataset of selected studies gathers full length articles that focus on firm-level business activity and management decisions in international contexts and show a specific focus on NC/CD.
} 
interpret the field as a result of their experience. In fact, to fully understand the structure of any field of study, qualitative/subjective analyses must be complemented with quantitative/objective ones that prevent any personal biases of the researchers and provide objectivity and reliability of results $^{2}$ (Acedo and Casillas, 2005).

The purpose of this article is to perform a comprehensive quantitative analysis of the relevance of NC/CD in IB literature. We use the same set of articles selected by LVG $(2015)^{3}$ in order to complement and improve LVG's qualitative findings. We contribute to the IB literature by providing insights on (I) faculty and institutional research productivity and performance; (II) articles, institutions, and scholars' influence in the contents of the field and its research agenda; and (III) the body of literature that has exerted the greatest impact on this set of selected articles. We conduct a series of bibliometric analyses that rely on the application of quantitative methods to a sample of articles (Pritchard, 1969). Examining the use of documents and publications patterns (Diodato,1994; Hawkins, 1977) within existing literature on a topic permits measuring, describing, and evaluating scientific publications (Hood and Wilson, 2001; Moed et al. 1995) and provides a monitoring device for university research management and even science policy. Thus we offer a quantitative, arguably objective, depiction of the field of study which may contribute to track academic advancements and steer future research endeavors. The bibliometric techniques used in this paper are productivity rankings (for both scholars and institutions), citation analysis, study of collaborative research trends, and analysis of the knowledge base.

\footnotetext{
${ }^{2}$ See Lahiri and Kumar (2012) for an exhaustive appraisal of the relevance of quantitative reviews on a wide variety of disciplinary areas.

${ }^{3}$ An appendix gathering the 265 articles used in LVG (2015) is available in the on-line version of the article published in the journal's website.
} 
The article is organized as follows: first, we present the analysis of most prolific and productive authors and institutions. Then the citation analysis identifying most influential works, authors, and institutions is presented. The following section explores the collaborative research trends within the field. Finally, the analysis of the knowledge base is performed and its results are depicted. The last section displays main conclusions, reflections and limitations.

\section{PRODUCTIVITY RANKINGS}

Productivity rankings inform about the most prolific and productive authors/institutions within the field. In order to provide a comprehensive overview, we rely on the total and adjusted number of articles published by authors and institutions, their length, and their performance in terms of 9 different journal metrics. In order to build these rankings, data relative to each article was collected and categorized by author/s, authors' institutional affiliation at the time of publication, institutions' host countries, year, journal, article length, and journals' performance in the year of publication.

\section{Absolute and adjusted productivity of authors and institutions: number of published articles}

Paper counts are the most basic bibliometric measure; nevertheless, they are a first approach to compare the productivity and volume of research among scholars and institutions. A high number of publications in top journals indicates that a scholar/institution has been prolific and successful in generating high quality research within the field and high visibility output (Peng and Zhou (2006), as well as in submitting her/his research to the critical review of fellow researchers and gaining their approval (Ramos-Rodríguez and Ruíz-Navarro (2004). These scholars/institutions may hold considerable influence on the direction of future research and should receive special credit for facilitating advancement of knowledge within this area. In other words, this analysis provides insights into the state of scholarship in this subfield by identifying scholars and institutions driving the research contexts and agenda. This is a particularly interesting issue if we 
take into account the increasing number of researchers and institutions that focus their attention on the IB field. Additionally, developing these rankings is useful for comparative purposes across different sub-fields or areas within the IB field, especially in verifying consistency in research productivity, as well as for analyzing the changing geographical scope of the institutions driving the research agenda.

Therefore, our first productivity measure gathers information about the number of published articles by scholars and institutions. For each of the 265 articles within the database we recorded information about the authors, their institutional affiliation at the time of publication, and the home country of each individual institution —we considered both academic and non-academic institutions. For each individual author/institution we considered both full (or total) and fractional (or adjusted) counting (Glänzel, 2001). The total or raw number of contributions gathers the absolute number of articles in which each author/institution is involved (regardless of the total number of co-authors in the article), while the adjusted contribution takes into account the number of different co-authors in an article. The adjustment process considers that an author contributes more, individually, with a single author article than with a multi-author article. Therefore, it recognizes, and adjusts for, that portion of the article attributable to the scholar/institution alone (Inkpen and Beamish, 1994; Knight et al. 2000). Following prior research (e.g.: Knight et al., 2000; Kumar and Kundu 2004; Quer et al. 2007, Treviño et al., 2010): (I) if an article was co-authored by more than one author from the same institution, the institution was credited with one appearance (total or adjusted) per author, (II) if an author listed a multiple institutional affiliation ${ }^{4}$, full credit (total or adjusted) was given to each institution; and (III) no distinction was made regarding the order of appearance of scholars.

\footnotetext{
${ }^{4}$ A slight $3 \%$ of the total amount of authors included in the database showed a multiple affiliation.
} 
A total of 523 different authors and 304 institutions are involved in the 265 selected articles. More than $80 \%$ of the authors and $55 \%$ of the academic institutions contributed only one article (absolute counting). Although the result for academic institutions is fairly consistent with Lotka's law ${ }^{5}$, the percentage of scholars contributing only once is quite higher than expected. Both authors and institutions counting adjust quite well to the square root $\operatorname{law}^{6}$, so that a relative low number of scholars/institutions (i.e.: 31 authors and 14 institutions) is involved in more than half of the total amount of articles. These results point to (I) a wide variety of researchers working in the area, but only a few being able to consistently publish in the selected top journals, (II) the existence of intra-university clusters of researchers working in the field, and (III) a high concentration ratio of authors and institutions that work in a persistent way in the field.

Tables 1 and 2 report the most prolific individual and institutional contributors to the field.

\footnotetext{
5 The first developemnt of this law was placed by Lotka in 1926 and it states that (I) the number (of authors) making $n$ contributions is about $1 / n^{2}$ of those making one and (II) the proportion of all contributors that makes a single contribution is about 60 per cent. See Glänzel and Schubert (1985) for an exhaustive review of the law contents and later updates.

${ }^{6}$ Formerly placed by the De Solla in 1963, the square root law states that half of the scientific papers within a field/topic/area are contributed by the top square root of the total number of scientific authors - see Glänzel and Schubert (1985) for a review and updates.
} 
Table 1. Most prolific authors

\begin{tabular}{|c|c|c|c|c|c|}
\hline Rank* & Author & $\begin{array}{c}\text { Adjusted } \\
\text { contributions/Total } \\
\text { contributions }\end{array}$ & Rank* & Author & $\begin{array}{c}\text { Adjusted } \\
\text { contributions/Total } \\
\text { contributions }\end{array}$ \\
\hline 1 & Luo, Yadong & $8.58 / 11$ & $16-23$ & Marshall, R. Scott & $1.5 / 2$ \\
\hline 2 & Slangen, Arjen & $3.58 / 7$ & $16-23$ & Mayrhofer, Ulrike & $1.5 / 2$ \\
\hline 3 & Ellis, Paul & $3 / 3$ & $16-23$ & Nielsen, Bo Bernhard & $1.5 / 2$ \\
\hline 4 & Griffith, David A. & $2.9 / 7$ & $16-23$ & Peltokorpi, Vesa & $1.5 / 2$ \\
\hline 5 & Shenkar, Oded & $2.41 / 7$ & $16-23$ & Tsang, Eric W. K. & $1.5 / 2$ \\
\hline 6 & Cavusgil, S. Tamer & $2.16 / 8$ & 24 & Vaara, Eero & $1.41 / 4$ \\
\hline 7 & Brock, David M. & $2.08 / 4$ & $25-27$ & Dikova, Desislava & $1.33 / 2$ \\
\hline 8 & Reus, Taco H. & $2 / 3$ & 25-27 & Hennart, Jean-Francois & $1.33 / 3$ \\
\hline 9 & Richards, Malika & $2 / 4$ & 25-27 & Janssens, Maddy & $1.33 / 2$ \\
\hline 10 & Schlegelmilch, Bodo B. & $1.83 / 4$ & 28-29 & Gong, Yaping & $1.25 / 2$ \\
\hline 11-14 & Demirbag, Mehmet & $1.66 / 5$ & 28-29 & Lin, Xiaohua & $1.25 / 3$ \\
\hline 11-14 & Glaister, Keith W. & $1.66 / 5$ & 30-33 & Hutzschenreuter, Thomas & $1.16 / 3$ \\
\hline 11-14 & Stahl, Günter K. & $1.66 / 5$ & 30-33 & Malhotra, Shavin & $1.16 / 3$ \\
\hline 11-14 & Tatoglu, Ekrem & $1.66 / 5$ & 30-33 & Sinkovics, Rudolf R. & $1.16 / 4$ \\
\hline 15 & Ambos, Borjn & $1.53 / 3$ & 30-33 & Sivakumar, K. & $1.16 / 3$ \\
\hline $16-23$ & Caligiuri, Paula M. & $1.5 / 2$ & 34 & Wang, Cheng Lu & $1.08 / 3$ \\
\hline 16-23 & Dow, Douglas & $1.5 / 3$ & $35-65$ & \multicolumn{2}{|c|}{31 authors whose score is 1 (adjusted contribution) } \\
\hline 16-23 & Drogendijk, Rian & $1.5 / 3$ & & & \\
\hline
\end{tabular}

*Ranked by adjusted number of contributions. 
As shown in Table 1, Yadong Luo, Arjen Slangen, Oded Shenkar and David Griffith are among the top 5 most prolific authors considering both raw and adjusted counting, contributing more than 2.4 times (adjusted contribution) to our selected list. Paul Ellis and S. Tamer Cavusgil are among the top five when considering only adjusted or total contribution respectively ${ }^{7}$, but they show a different profile: while Ellis contributes with 3 single authored articles to the database, Cavusgil contributes with 8 articles, but participates repeatedly in intra and extramural research teams. As shown in Table 1, large differences exist when comparing total and adjusted contributions of most prolific authors pointing, once again, to the relevance of collaborative research. Noteworthy is the collaboration among most prolific researchers (e.g.: Luo and Shenkar, Griffith and Cavusgil). The magnitude of contribution of leading authors can best be viewed in comparison to the average appearance of 0.51 . Furthermore, taking into account their total number of contributions, the wide spectrum of journals they have published in, as well as existing distinctions among these journals in terms of aim and scope, we can affirm that some of these prolific authors (e.g.: Luo, Shenkar) have reached a particularly extensive and diverse audience.

\footnotetext{
${ }^{7}$ Luo has been identified as the most prolific author in different studies relative to the entire IB field (Lahiri and Kumar, 2012; Xu et al. 2008), as well as studies relative to a particular sub-field or area — e.g.: the study by Quer et al. (2007) relative to business and management in China. Cavusgil, Elllis, Griffith, Shenkar, and Tsang are also among the most prolific authors (adjusted counting) identified in $\mathrm{Xu}$ et al. (2008).
} 
Table 2. Most prolific institutions

\begin{tabular}{|c|c|c|c|c|c|}
\hline Rank* & Institution & $\begin{array}{c}\text { Adjusted } \\
\text { contributions/Total } \\
\text { contributions }\end{array}$ & Rank* & Institution & $\begin{array}{c}\text { Adjusted } \\
\text { contributions/Total } \\
\text { contributions }\end{array}$ \\
\hline 1 & U. Miami & $8.92 / 12$ & $22-24$ & INSEAD & $2.5 / 7$ \\
\hline 2 & Erasmus U. & $7.16 / 13$ & $22-24$ & U. Groningen & $2.5 / 5$ \\
\hline 3 & Vienna U. Economics and Business Administration & $5.16 / 12$ & $22-24$ & U. Minessota & $2.5 / 5$ \\
\hline 4 & U. London & $4 / 9$ & 25 & Ben-Gurion U. & $2.25 / 3$ \\
\hline 5 & Tilburg U. & $3 / 10$ & 26 & U. Auckland & $2.17 / 5$ \\
\hline 6 & Rutgers U. & $3.85 / 11$ & 27 & U. Pennsylvania & $2.02 / 6$ \\
\hline 7 & Hanken School of Economics & $3.71 / 13$ & $28-35$ & Chinese U. Hong Kong & $2 / 7$ \\
\hline 8 & U. South Carolina & $3.67 / 8$ & $28-35$ & U. Alicante & $2 / 6$ \\
\hline 9 & Michigan State U. & $3.5 / 12$ & $28-35$ & Hong Kong U. Science \& Technology & $2 / 5$ \\
\hline $10-11$ & U. Sheffield & $3.33 / 10$ & $28-35$ & Loughborough U. & $2 / 5$ \\
\hline $10-11$ & WHU - Otto Beisheim School of Management & $3.33 / 8$ & $28-35$ & Stockholm U. & $2 / 5$ \\
\hline 12 & Uppsala U. & $2.87 / 7$ & $28-35$ & U. Leeds & $2 / 5$ \\
\hline $13-14$ & U. the West of England & $3 / 5$ & $28-35$ & Katholicka Universiteit Leuven & $2 / 4$ \\
\hline $13-14$ & Hong Kong Polytechnic U. & $3 / 3$ & $28-35$ & U. Vaasa & $2 / 4$ \\
\hline 15 & U. Western Ontario & $2.83 / 8$ & $36-39$ & Hong Kong Baptist U. & $1.83 / 8$ \\
\hline 16 & U. Amsterdam & $2.77 / 8$ & $36-39$ & U. Oklahoma & $1.83 / 6$ \\
\hline $17-18$ & Ohio State U. & $2.67 / 8$ & $36-39$ & U. New South Wales & $1.83 / 3$ \\
\hline $17-18$ & Monash U. & $2.67 / 7$ & $40-42$ & National Chengchi U. & $1.75 / 5$ \\
\hline 19 & Texas A\&M U. & $2.62 / 6$ & $40-42$ & Pennsylvania State U. & $1.75 / 4$ \\
\hline 20 & U. Texas at Dallas & $2.58 / 5$ & $40-42$ & U. North Carolina & $1.75 / 4$ \\
\hline 21 & U. Melbourne & $2.54 / 6$ & $43-59$ & \multicolumn{2}{|c|}{17 institutions whose score is 1.5 or higher (adjusted contribution) } \\
\hline
\end{tabular}

\footnotetext{
*Ranked by adjusted number of contributions.
}

U.: University/University of 
The ranking of most prolific institutions is led by the University of Miami (USA), the Erasmus University of Rotterdam (Netherlands), and the Vienna University of Economics and Business Administration (Austria) both in terms of full and adjusted contribution. Actually, the set of universities included in the top-10 are the same in both total and adjusted rankings, gathering six European universities and four US universities. While the outstanding position of some of these institutions is tightly linked to individual prolific authors (e.g.: University of Miami-Yadong Luo), wider groups of authors contribute to the positioning of other academic institutions (e.g.: Erasmus University-6 authors: Vienna University-5 authors). A wider exploration of the geographic patterns shows that European institutions dominate this research area or sub-field $40 / 45 \%$ (total/adjusted contribution, respectively) of the top-50 academic universities are European-, while the US remains at a second place-37/32\% representation. Conversely, the presence of institutions from Asia-Pacific remains at about $20 \%$ in both rankings with no institutions within the top-10. These results point to a wide internationalization of the knowledge creation process within this sub-field, as institutions from around the globe are involved in this body of research. Our findings corroborate only partially previous studies: although the internationalization of knowledge creation was already predicted by Inkpen and Beamish (1994) ${ }^{8}$ and later corroborated by Lahiri and Kumar (2012), Treviño et al. (2010), and Xu et al. (2008) ${ }^{9}$, these studies show a predominant role of North American institutions and an emerging and/or far second (even third) place of European institutions. In addition, the most recent studies point to institutions coming from Asia-Pacific as a growing force that is not corroborated in our study.

\footnotetext{
8 They found a slight increase in the weight of non-US institutions when comparing their results with those of a previous study by Morrison and Inkpen (1991).

9 These studies are not centered in any particular issue or subject within the IB field.
} 
Conversely, our findings point to the relevance of European institutions in driving the research agenda within this particular sub-field in the analyzed period.

\section{Absolute and adjusted productivity of authors and institutions based on journals' performance}

Journal metrics play a central role in most performance evaluations of research outputs as they have become a highly used way to rank scientific journals. As stated in Glänzel and Moed (2002), journal metrics are designed to assess the significance and performance of individual journals, their role and position in the international formal communication network, and their quality or prestige as perceived by scholars. Different metrics exist, each of them calibrated to take into account specific features and focused on a particular (therefore, limited) perspective. Bibliometricians agree on the complex and multi-dimensional nature of journal performance (Moed et al., 2012). As a consequence, no single metric can effectively capture the entire spectrum of research performance; using Moed's (2010, p. 274) words "there is no single perfect indicator of journal performance".

In order to provide a wide perspective of authors and institutions' productivity based on journals performance, and following suggestions in the White Paper on the Evolution of Journals Assessment, our analysis is based on nine different metrics whose respective methodologies, data sources, advantages, and drawbacks have been widely discussed in existing literature ${ }^{10}$. In particular, we have based on the metrics calculated and published by Thomson Reuters -i.e.: Journal Citations Reports (JCR)—, Eigenfactor Organization, and Scopus: JCR Annual-impact factor (JCR), JCR Annual-impact factor without journal self-citation (JCR-wsc), JCR 5-year

\footnotetext{
${ }^{10}$ See, among others, Bergstrom (2007), Bergstrom et al. (2008). Colledge et al. (2010), González-Pereira et al. (2010), Guerrero-Bote and Moya-Anegón (2012), Moed et al. (2012), Sicilia et al. (2011), and Waltman et al. (2013).
} 
impact factor (JCR-5), JCR Immediacy index (JCR-im), Eigenfactor Score (EgS), Article Influence Score (AI), Impact per Publication (IPP), Source Normalized Impact per Paper (SNIP), and Scimago Journal Rank (SJR) — see Table 3 for an overview of these metrics. 
Table 3. Overview of journal metrics

\begin{tabular}{|c|c|c|c|c|}
\hline Metric & & Publisher & Description & Data Source \\
\hline Journal Citation Report & JCR & Thomson Reuters & $\begin{array}{l}\text { The frequency with which the average article in a journal } \\
\text { has been cited in a } 2 \text {-year period of time }\end{array}$ & Thomson Reuters \\
\hline $\begin{array}{l}\text { Journal Citation Report Immediacy } \\
\text { Index }\end{array}$ & JCR-Im & Thomson Reuters & $\begin{array}{l}\text { The frequency with which the average article in a journal } \\
\text { has been cited in the same year that it has been published }\end{array}$ & Thomson Reuters \\
\hline $\begin{array}{l}\text { Journal Citation Report without self- } \\
\text { citation }\end{array}$ & JCR-wsc & Thomson Reuters & $\begin{array}{l}\text { The frequency with which the average article in a journal } \\
\text { has been cited in a } 2 \text {-year period of time. Citations coming } \\
\text { from the same journal are not considered }\end{array}$ & Thomson Reuters \\
\hline 5 year Journal Citation report* & JCR-5 & Thomson Reuters & $\begin{array}{l}\text { The frequency with which the average article in a journal } \\
\text { has been cited in a } 5 \text {-year period of time }\end{array}$ & Thomson Reuters \\
\hline Eigenfactor Score & $\mathrm{EgS}$ & $\begin{array}{l}\text { Eigenfactor } \\
\text { Organization }\end{array}$ & $\begin{array}{l}\text { The frequency with which the average article in a journal } \\
\text { has been cited in a } 5 \text {-year period of time. Citing journals } \\
\text { are weighted depending on their influence }\end{array}$ & Thomson Reuters \\
\hline Article Influence & AI & $\begin{array}{l}\text { Eigenfactor } \\
\text { Organization }\end{array}$ & $\begin{array}{l}\text { Ratio between the journal } \mathrm{EgS} \text { and the normalized fraction } \\
\text { of articles published by this journal }\end{array}$ & Thomson Reuters \\
\hline Scimago Journal Rank & SJR & Scopus & $\begin{array}{l}\text { The frequency with which the average article in a journal } \\
\text { has been cited in a } 3 \text {-year period of time. Citing journals } \\
\text { are weighted depending on their influence and the subject } \\
\text { field }\end{array}$ & Scopus** \\
\hline Impact per Publication & IPP & Scopus & $\begin{array}{l}\text { The frequency with which the average article in a journal } \\
\text { has been cited in a } 3 \text {-year period of time }\end{array}$ & Scopus \\
\hline Source Normalized Impact per Paper & SNIP & Scopus & $\begin{array}{l}\text { The journal IPP normalized for the citations in the subject } \\
\text { field }\end{array}$ & Scopus \\
\hline
\end{tabular}

\footnotetext{
*Only available for articles published from 2007. ** Scopus database gathers a higher number of journals than Thomson Reuters.
} 
To assess institutions and authors' productivity the following process was followed: as a first step and for each of the 265 articles, we considered the nine different journal metrics in the year that the article was published. Second, we weighted each of these metrics by the adjusted number of authors and the standardized article's length - the total number of pages per article was standardized based on a conversion factor using the average number of pages of the articles published in the Journal of International Business Studies. Then, a cumulative value was estimated for each of the 523 authors by adding the scores relative to all the articles in which the author was involved. The final output is an author's cumulative productivity value for each of the nine different metrics considered. The same process was applied in order to assess the productivity of the 304 institutions. Tables 4 and 5 present these cumulative values for the most productive authors and institutions respectively.

As said before, each metric aims at favoring a particular perspective; therefore, rankings differ from one another. Although correlations exist, they are far from perfect. Focusing on the leadership positions in each ranking, we find 2 authors that keep consistently among the top-10 regardless of the metric considered: Luo Yadong and Eero Vaara ${ }^{11}$. As they keep this position even in the JCR-5 that is only calculated for articles published after 2007, we can affirm that their leadership keeps consistent all along the analyzed period. Gunter Stahl keeps within this top-10 group for all metrics except JCR-5, pointing to a leadership position similar to that of the former two, but in the first sub-period (2000-2006). Conversely, other authors (e.g. Johannes C. Voll) enter the top-10 only for JCR-5, pointing to the opposite situation. Oded Shenkar and Dan V.

\footnotetext{
${ }^{11}$ Up to 9 of our top-50 most productive scholars (JCR) are also among the most productive authors in the entire IB field in the period 1996-2008 as shown in the study by Treviño et al. (2010).
} 
Caprar keep a consistent prominent position except for immediacy index; while Alain Verbeke, Julia Eiche, Christian Schwens, and Ruediger Kabst enter this top-10 list only when considering the immediacy degree of the citation process, pointing to cutting-the-edge research quickly acknowledged and cited by colleagues. Nevertheless, it must be noted that the immediacy index is biased by the number of issues per year published by the journal: articles in journals that publish a high number of issues per year (e.g.: Strategic Management Journal, Journal of Management Studies) are more likely to be cited within the same year than articles published in journals that publish a low number of issues per year (e.g.: Academy of Management Review, Journal of World Business).

As shown in Table 4, some authors enter this top-10 selected group when the citing journals influence is considered (e.g.: Yaping Gong, Craig Crossland, Donald Hambrick, Albert Cannella, Mohan Subramanian), while other authors enter this group when the data source is broadened (e.g.: David Griffith, Taco Reus). Arjen Slangen, Riika Sarala, Paul Ellis, S. Tamer Cavusgil, and Thomas Hutzschenreuter are also consistently among the top-10 in terms of productivity based on journals metrics. 
Table 4. Most productive authors based on journals performance

\begin{tabular}{|c|c|c|c|c|c|c|c|c|c|c|c|c|c|c|c|c|c|}
\hline \multicolumn{2}{|l|}{ JCR } & \multicolumn{2}{|l|}{ JCR-im } & \multicolumn{2}{|c|}{ JCR-wsc } & \multicolumn{2}{|l|}{ EgS } & \multicolumn{2}{|l|}{ AI } & \multicolumn{2}{|l|}{ SJR } & \multicolumn{2}{|l|}{ SNIP } & \multicolumn{2}{|l|}{ IPP } & \multicolumn{2}{|l|}{ JCR-5* } \\
\hline Luo, Y. & 18.39 & Luo, Y. & 3.01 & Luo, Y. & 15.89 & Luo, Y. & 0.09 & Luo, Y. & 19.07 & Luo, Y. & 29.48 & Luo, Y. & 20.59 & Luo, Y. & 24.53 & Luo, Y. & 7.06 \\
\hline Vaara, E. & 5.58 & Hutzschenreuter, T. & 1.15 & Vaara, E. & 4.46 & Gong, Y. & 0.02 & Shenkar, O. & 2.82 & Shenkar, O. & 5.61 & Vaara, E. & 4.30 & Vaara, E. & 6.62 & Vaara, E. & 6.66 \\
\hline Stahl, G. & 4.74 & Voll, J.C. & 1.11 & Stahl, G. & 3.96 & Stahl, G. & 0.02 & Vaara, E. & 2.62 & Vaara, E. & 5.38 & Ellis, $P$. & 4.07 & Slangen, A. & 6.20 & Caprar, D.V. & 5.92 \\
\hline Slangen, A. & 4.67 & Vaara, E. & 1.05 & Shenkar, O. & 3.83 & Vaara, E. & 0.02 & Gong, Y. & 2.59 & Stahl, G. & 5.10 & Slangen, A. & 4.00 & Ellis, $\mathrm{P}$. & 6.03 & Sarala, R. & 5.76 \\
\hline Sarala, R. & 4.43 & Verbeke, A. & 0.95 & Slangen, A. & 3.63 & Shenkar, O. & 0.02 & Ellis, P. & 2.35 & Ellis, $\mathrm{P}$. & 4.55 & Shenkar, O. & 3.69 & Stahl, G.K. & 5.86 & Ellis, P. & 5.21 \\
\hline Ellis, P. & 4.32 & Sarala, R. & 0.95 & Sarala, R. & 3.48 & Caprar, D.V. & 0.01 & Slangen, A. & 2.30 & Slangen, A. & 4.51 & Stahl, G.K. & 3.68 & Reus, $\mathrm{T}$. & 5.61 & Shenkar, O. & 5.02 \\
\hline Shenkar, O. & 4.21 & Stahl, G. & 0.86 & Ellis, P. & 3.30 & Crossland, C. & 0.01 & Stahl, G. & 2.27 & Caprar, D.V. & 4.21 & Cavusgil, S.T. & 3.51 & Cavusgil, S.T. & 5.06 & Slangen, A. & 5.00 \\
\hline Cavusgil, S.T. & 4.07 & Eiche, J & 0.83 & Hutzschenreuter, T. & 3.06 & Hambrick, D.C. & 0.01 & Hutzschenreuter, T. & 2.15 & Cavusgil, S.T. & 4.05 & Hutzschenreuter, T. & 2.97 & Caprar, D.V. & 4.98 & Hutzschenreuter, T. & 4.84 \\
\hline Caprar, D.V. & 3.92 & Kabst, R & 0.83 & Caprar, D.V. & 3.06 & Cannella, A.A. & 0.01 & Caprar, D.V. & 1.94 & Sarala, R. & 3.96 & Caprar, D.V. & 2.91 & Shenkar, O. & 4.88 & Voll, J.C. & 4.84 \\
\hline Hutzschenreuter, T. & 3.81 & Schwens, C & 0.83 & Cavusgil, S.T. & 3.03 & Subramaniam, M. & 0.01 & Cavusgil, S.T. & 1.90 & Gong, Y. & 3.91 & Griffith, D.A. & 2.85 & Sarala, R. & 4.82 & Cavusgil, S.T. & 4.71 \\
\hline Sinkovics, R.R. & 3.77 & Caprar, D.V. & 0.81 & Sinkovics, R.R. & 2.79 & $\mathrm{Yu}, \mathrm{T}$. & 0.01 & Sarala, R. & 1.81 & Crossland, C. & 3.89 & Sarala, R. & 2.76 & Hutzschenreuter, $\mathrm{T}$. & 4.54 & Reus, $\mathrm{T}$. & 4.69 \\
\hline Voll, J.C. & 3.42 & Shenkar, O. & 0.72 & Voll, J.C. & 2.76 & Caligiuri, P. & 0.01 & Cannella, A.A. & 1.79 & Hambrick, D.C. & 3.89 & Sinkovics, R.R. & 2.68 & Sinkovics, R.R. & 4.51 & Sinkovics, R.R. & 4.53 \\
\hline Reus, T. & 3.31 & Slangen, A. & 0.72 & Reus, $\mathrm{T}$. & 2.53 & Sarala, R. & 0.01 & Subramaniam, M. & 1.79 & Reus, T. & 3.72 & Hennart, H.F. & 2.51 & Voll, J.C. & 3.91 & Stahl, G. & 4.18 \\
\hline Jean R.J.B. & 3.15 & Björkman, I. & 0.58 & Cannella, A.A. & 2.31 & Slangen, A. & 0.01 & $\mathrm{Yu}, \mathrm{T}$. & 1.79 & Hutrschemerter, $\mathrm{T}$. & 3.58 & Voll, J.C. & 2.50 & Jean, R.J.B. & 3.56 & Crossland, C. & 3.98 \\
\hline Kang, J.K. & 2.77 & Cavusgil, S.T. & 0.57 & Subramaniam, M. & 2.31 & Hutzschenreuter, T. & 0.01 & Crossland, C. & 1.79 & Cannella, A.A. & 3.51 & Reus, T. & 2.48 & Demirbag, M. & 3.51 & Hambrick, D.C. & 3.98 \\
\hline Kim, J.M. & 2.77 & Brouthers, K. & 0.55 & Yu, T. & 2.31 & Delios, A. & 0.01 & Hambrick, D.C. & 1.79 & Subramaniam M & 3.51 & Gong, Y. & 2.45 & Glaister, K.W. & 3.51 & Kang, J.K. & 3.67 \\
\hline Cuypers, I. & 2.65 & Fee, A. & 0.53 & Jean, R.J.B. & 2.28 & Henisz, W.J. & 0.01 & Voll, J.C. & 1.63 & Yu, T. & 3.51 & Demirbag, M. & 2.27 & Tatoglu, E. & 3.51 & Kim, J.M. & 3.67 \\
\hline Martin, X. & 2.65 & Gray, S.J. & 0.53 & Crossland, C. & 2.18 & Cavusgil, S.T. & 0.01 & Hennart, H.F. & 1.62 & Sinkovics, R.R. & 3.30 & Glaister, K.W. & 2.27 & Hennart, H.F. & 3.42 & Jean, R.J.B. & 3.62 \\
\hline Hennart, H.F. & 2.57 & Bamossy, G.J. & 0.51 & Hambrick, D.C. & 2.18 & Ellis, P. & 0.01 & Sinkovics, R.R. & 1.51 & Voll, J.C. & 3.15 & Tatoglu, E. & 2.27 & Drogendijk, R. & 3.31 & Cannella, A.A. & 3.55 \\
\hline Cannella, A.A. & 2.49 & Sinkovics, R.R. & 0.50 & Gong, Y. & 2.17 & Voll, J.C. & 0.01 & $\mathrm{Kim}, \mathrm{K}$ & 1.50 & Hennart, H.F. & 3.11 & Katsikeas, C.S. & 2.20 & Dow, D. & 3.25 & Subramaniam, M. & 3.55 \\
\hline Subramaniam, M. & 2.49 & Kang, J.K. & 0.48 & Kang, J.K. & 2.06 & Kim, K. & 0.01 & Yeheske, O. & 1.48 & Yeheske, O. & 2.91 & Skarmeas, D. & 2.20 & Floyd, S.W. & 3.20 & $\mathrm{Yu}, \mathrm{T}$. & 3.55 \\
\hline $\mathrm{Yu}, \mathrm{T}$. & 2.49 & Kim, J.M. & 0.48 & Kim, J.M. & 2.06 & Floyd, S.W. & 0.01 & Demirbag, M. & 1.42 & Kim, K. & 2.88 & Dow, D. & 2.19 & Barkema, H. & 3.19 & Cuypers, I. & 3.51 \\
\hline Dikova, D. & 2.44 & Uhlenbruk, K. & 0.47 & Hennart, H.F. & 2.04 & Brouthers, $\mathrm{K}$. & 0.01 & Glaister, K.W. & 1.42 & Barkema, $\mathrm{H}$. & 2.77 & Floyd, S.W. & 2.17 & Crossland, C. & 3.17 & Martin, X. & 3.51 \\
\hline Crossland, C. & 2.40 & Dikova, D. & 0.47 & Yeheske, O. & 2.04 & Lepak, D. & 0.01 & Tatoglu, E. & 1.42 & Katsikeas, C.S. & 2.76 & Barkema, H. & 2.15 & Hambrick, D.C. & 3.17 & $\mathrm{Kim}, \mathrm{K}$ & 3.48 \\
\hline Hambrick, D.C. & 2.40 & Cuypers, I. & 0.46 & Björkman, I. & 1.98 & Takeuchi, R. & 0.01 & Dow, D. & 1.42 & Skarmeas, D. & 2.76 & Marshall, R.S. & 2.14 & Knippenberg, D. & 3.06 & Aybar, B. & 3.46 \\
\hline Katsikeas, C.S. & 2.39 & Martin, $X$ & 0.46 & Cuypers, I. & 1.97 & Yun, S. & 0.01 & Larimo, J. & 1.29 & Floyd, S.W. & 2.58 & Drogendijk, R. & 2.12 & Tröster, C. & 3.06 & Ficici, A. & 3.46 \\
\hline Skarmeas, D. & 2.39 & Malhotra, S. & 0.44 & Martin, $X$. & 1.97 & Knippenberg, D. & 0.01 & Reus, T. & 1.29 & Knippenberg, D. & 2.57 & Schlegelmilch, BB. & 2.08 & Griffith, D.A. & 3.04 & Chang, K. & 3.30 \\
\hline Gong, Y. & 2.37 & Sivakumar, K. & 0.44 & Demirbag, M. & 1.74 & Tröster, C. & 0.01 & Delios, A. & 1.26 & Tröster, C. & 2.57 & Crossland, C. & 2.06 & Björkman, I. & 2.99 & Chari, M. & 3.30 \\
\hline Dow, D. & 2.35 & Yeheske, O. & 0.42 & Glaister, K.W. & 1.74 & Chen, G. & 0.01 & Henisz, W.J. & 1.26 & Demirbag, M. & 2.49 & Hambrick, D.C. & 2.06 & Gong, Y. & 2.94 & Johanson, J. & 3.30 \\
\hline Björkman, I. & 2.34 & Jean, R.J.B. & 0.40 & Tatoglu, E. & 1.74 & Farh, C. & 0.01 & Chen, G. & 1.24 & Glaister, K.W. & 2.49 & Jean, R.J.B. & 2.05 & Nielsen, B. & 2.93 & Vahlne, J.E. & 3.30 \\
\hline Nielsen, B. & 2.31 & Ellis, $\mathrm{P}$. & 0.40 & Dikova, D. & 1.72 & Kirkman, B. & 0.01 & Farh, C. & 1.24 & Tatoglu, E. & 2.49 & Ragozzino, R. & 2.01 & Katsikeas, C.S. & 2.91 & Knippenberg, D. & 3.28 \\
\hline Aybar, B. & 2.28 & Beugelsdijk, S. & 0.35 & Barkema, H. & 1.70 & Tangirala, $\mathrm{S}$. & 0.01 & Kirkman, B. & 1.24 & Jean, R.J.B. & 2.47 & Malhotra, S. & 1.99 & Skarmeas, D. & 2.91 & Tröster, C. & 3.28 \\
\hline Ficici, A. & 2.28 & Demirbag, M. & 0.35 & Griffith, D.A. & 1.63 & Hennart, H.F. & 0.01 & Tangirala, S. & 1.24 & Björkman, I. & 2.43 & Sivakumar, K. & 1.99 & Kang, J.K. & 2.86 & Björkman, I. & 3.10 \\
\hline Demirbag, M. & 2.20 & Glaister, K.W. & 0.35 & Katsikeas, C.S. & 1.63 & Kostova, T. & 0.01 & Floyd, S.W. & 1.24 & Delios, A. & 2.36 & Brouthers, K. & 1.96 & Kim, J.M. & 2.86 & Barkema, H. & 3.07 \\
\hline Glaister, K.W. & 2.20 & Tatoglu, E. & 0.35 & Skarmeas, D. & 1.63 & Yeheske, O. & 0.01 & Kostova, $\mathrm{T}$. & 1.20 & Henisz, W.J. & 2.36 & Björkman, I. & 1.89 & Malhotra, S. & 2.85 & Abdi, M. & 2.98 \\
\hline Tatoglu, E. & 2.20 & Barinaga, E. & 0.33 & Beugelsdijk, S. & 1.58 & Abdi, M. & 0.01 & Jean, R.J.B. & 1.19 & Chen, G. & 2.36 & Knippenberg, D. & 1.86 & Abdi, M. & 2.79 & Aulakh, P. & 2.98 \\
\hline Griffith, D.A. & 2.19 & Knippenberg, D. & 0.32 & Verbeke, A. & 1.56 & Aulakh, P. & 0.01 & Nielsen, B. & 1.16 & Farh, C. & 2.36 & Tröster, C. & 1.86 & Aulakh, P. & 2.79 & Lamont, B.T. & 2.97 \\
\hline Chang, K. & 2.17 & Tröster, C. & 0.32 & Floyd, S.W. & 1.54 & Brannen, M.Y. & 0.01 & Brouthers, K. & 1.16 & Kirkman, B. & 2.36 & Larimo, J. & 1.86 & Dikova, D. & 2.77 & Yeheske, O. & 2.84 \\
\hline Chari, M. & 2.17 & Dow, D. & 0.32 & Knippenberg, D. & 1.51 & Salk, J.E. & 0.01 & Knippenberg, D. & 1.15 & Tangirala, S. & 2.36 & Nielsen, B. & 1.78 & Cuypers, I. & 2.73 & Floyd, S.W. & 2.69 \\
\hline Johanson, J. & 2.17 & Barkema, $\mathrm{H}$. & 0.32 & Tröster, C. & 1.51 & Vora, D. & 0.01 & Tröster, C. & 1.15 & Abdi, M. & 2.34 & Raz, A. & 1.76 & Martin, $X$. & 2.73 & Prashantham, S. & 2.69 \\
\hline
\end{tabular}

*Only for articles published from 2007 to 2012. 
Heterogeneity among institutional rankings is less striking than among authors' ones (Table 5). 3 European universities keep among the top-10 regardless of the metrics considered - the Erasmus University of Rotterdam (Netherlands), the Hanken School of Economics (Finland), and the WHU Otto Beisheim School of Management (Germany). The University of Miami (USA) also remains within the top-10 for all journal metrics except for the JCR-5 (only available for articles published in the last years of the analyzed period). The Tilburg University (Netherlands), the Rutgers University (USA), and the University of London (UK) also show particularly prominent positions. Diversity is found when dealing with the immediacy of citation, weighting citing journals influence or broadening the data source; for instance, the Giessen University (Germany), the Texas A\&M University (USA), the University of Groningen (Netherlands), and the University of Calgary (Canada) arise within the institutions publishing quickly cited research, the Hong Kong University of Sciences and Technology (Hong Kong), the Boston College (USA), and the University of Maryland (USA) remain within the top-10 when citing journals are weighted; and the Vienna University of Economics and Business (Austria) and the INSEAD (France) arise among the top-10 when the citing database is broader. Some academic institutions clearly improve their position when the analysis focuses only on the last sub-period of study, that is on JCR-5 (e.g.: Uppsala University-Sweden, the University of North Carolina-USA); while others (e.g. the University of Pennsylvania-USA) are among the top-10 in JCR and JCRws, but do not keep this position when considering the remaining metrics. 
Table 5. Most productive institutions based on journals performance

\begin{tabular}{|c|c|c|c|c|c|c|c|c|c|c|c|c|c|c|c|c|c|}
\hline \multicolumn{2}{|l|}{ JCR } & \multicolumn{2}{|l|}{ JCR-im } & \multicolumn{2}{|l|}{ JCR-wsc } & \multicolumn{2}{|l|}{ EgS } & \multicolumn{2}{|l|}{ AI } & \multicolumn{2}{|l|}{ SJR } & \multicolumn{2}{|l|}{ SNIP } & \multicolumn{2}{|l|}{ IPP } & \multicolumn{2}{|l|}{ JCR-5* } \\
\hline U. Miami & 18.95 & U. Miami & 3.07 & U. Miami & 16.42 & U. Miami & 0.10 & U. Miami & 19.07 & U. Miami & 30.01 & U. Miami & 20.98 & U. Miami & 25.33 & $\begin{array}{l}\text { Erasmus U. } \\
\text { Rotterdam }\end{array}$ & 15.18 \\
\hline $\begin{array}{l}\text { Erasmus U. } \\
\text { Rotterdam }\end{array}$ & 11.41 & U. Giessen & 2.50 & $\begin{array}{l}\text { Erasmus U. } \\
\text { Rotterdam }\end{array}$ & 9.02 & $\begin{array}{l}\text { Hong Kong U. } \\
\text { S\&T. }\end{array}$ & 0.03 & $\begin{array}{l}\text { Erasmus U. } \\
\text { Rotterdam }\end{array}$ & 5.79 & $\begin{array}{l}\text { Erasmus U. } \\
\text { Rotterdam }\end{array}$ & 13.53 & $\begin{array}{l}\text { Erasmus U. } \\
\text { Rotterdam }\end{array}$ & 12.06 & $\begin{array}{l}\text { Erasmus U. } \\
\text { Rotterdam }\end{array}$ & 17.98 & Hanken S.E. & 12.79 \\
\hline $\begin{array}{l}\text { WHU - Otto } \\
\text { Beisheim S.M. }\end{array}$ & 9.74 & $\begin{array}{l}\text { WHU - Otto } \\
\text { Beisheim S.M. }\end{array}$ & 2.44 & $\begin{array}{l}\text { WHU - Otto } \\
\text { Beisheim S.M. }\end{array}$ & 8.10 & Rutgers U. & 0.03 & $\begin{array}{l}\text { WHU - Otto } \\
\text { Beisheim S.M. }\end{array}$ & 5.11 & Hanken S.E. & 8.66 & $\begin{array}{l}\text { WHU - Otto } \\
\text { Beisheim S.M. }\end{array}$ & 7.82 & $\begin{array}{l}\text { WHU - Otto } \\
\text { Beisheim S.M. }\end{array}$ & 12.03 & $\begin{array}{l}\text { WHU - Otto } \\
\text { Beisheim S.M. }\end{array}$ & 12.36 \\
\hline Hanken S.E. & 9.70 & Hanken S.E. & 1.85 & Hanken S.E. & 8.05 & U. Maryland & 0.03 & U. Maryland & 4.54 & Tilburg U. & 8.55 & Hanken S.E. & 7.26 & Hanken S.E. & 11.79 & Tilburg U. & 9.18 \\
\hline Tilburg U. & 7.41 & $\begin{array}{l}\text { Erasmus U. } \\
\text { Rotterdam }\end{array}$ & 1.54 & Tilburg U. & 5.79 & $\begin{array}{l}\text { Erasmus U. } \\
\text { Rotterdam }\end{array}$ & 0.03 & Boston College & 4.25 & $\begin{array}{l}\text { WHU -Otto } \\
\text { Beisheim S.M. }\end{array}$ & 8.50 & Tilburg U. & 6.75 & Tilburg U. & 10.16 & Uppsala U. & 8.23 \\
\hline Rutgers U. & 7.10 & U. London & 1.24 & Rutgers U. & 5.43 & Boston College & 0.03 & Hanken S.E. & 4.15 & Rutgers U. & 8.48 & U. London & 6.65 & U. London & 9.12 & Texas A\&M U. & 7.86 \\
\hline U. London & 6.75 & Texas A\&M U. & 1.16 & U. London & 5.12 & INSEAD & 0.02 & Rutgers U. & 4.08 & U. Maryland & 8.12 & Rutgers U. & 6.24 & Texas A\&M U. & 8.82 & U. North Carolina & 7.57 \\
\hline U. Groningen & 6.31 & U. Groningen & 1.12 & Boston College & 4.97 & $\begin{array}{l}\text { WHU - Otto } \\
\text { Beisheim S.M. }\end{array}$ & 0.02 & $\begin{array}{l}\text { Hong Kong U. } \\
\text { S\&T. }\end{array}$ & 4.03 & Texas A\&M U. & 8.04 & Texas A\&M U. & 6.05 & Rutgers U. & 8.77 & U. Maryland & 7.50 \\
\hline $\begin{array}{l}\text { U. } \\
\text { Pennsylvania }\end{array}$ & 6.28 & U. Calgary & 1.02 & INSEAD & 4.96 & Texas A\&M U. & 0.02 & Texas A\&M U. & 3.78 & U. London & 7.88 & $\begin{array}{l}\text { Vienna U. E\&B } \\
\text { Administration }\end{array}$ & 5.91 & $\begin{array}{l}\text { Vienna U. E\&B } \\
\text { Administration }\end{array}$ & 8.47 & Boston College & 7.11 \\
\hline INSEAD & 6.12 & Tilburg U. & 1.00 & $\begin{array}{l}\text { U. } \\
\text { Pennsylvania }\end{array}$ & 4.76 & Hanken S.E. & 0.02 & Tilburg U. & 3.69 & Boston College & 7.75 & INSEAD & 5.50 & INSEAD & 8.00 & U. Groningen & 7.07 \\
\hline Uppsala U. & 6.00 & INSEAD & 0.99 & $\begin{array}{l}\text { Vienna U. E\&B } \\
\text { Administration }\end{array}$ & 4.69 & $\begin{array}{l}\text { U. } \\
\text { Pennsylvania }\end{array}$ & 0.02 & INSEAD & 3.32 & INSEAD & 7.41 & $\begin{array}{l}\text { U. Texas at } \\
\text { Dallas }\end{array}$ & 5.18 & Uppsala U. & 7.42 & U. Miami & 7.06 \\
\hline $\begin{array}{l}\text { Vienna U. E\&B } \\
\text { Administration }\end{array}$ & 5.62 & $\begin{array}{l}\text { U. } \\
\text { Pennsylvania }\end{array}$ & 0.98 & U. Groningen & 4.61 & Ohaio State U. & 0.02 & U. London & 3.21 & $\begin{array}{l}\text { Hong Kong U. } \\
\text { S\&T. }\end{array}$ & 7.04 & Uppsala U. & 4.75 & $\begin{array}{l}\text { U. Texas at } \\
\text { Dallas }\end{array}$ & 7.36 & Georgia State U. & 7.02 \\
\hline Texas A\&M U. & 5.58 & $\begin{array}{l}\text { U. North } \\
\text { Carolina at } \\
\text { Greensboro }\end{array}$ & 0.95 & Texas A\&M U. & 4.49 & Tilburg U. & 0.02 & Ohaio State U. & 3.09 & $\begin{array}{l}\text { Vienna U. E\&B } \\
\text { Administration }\end{array}$ & 6.96 & U. Sheffield & 4.54 & U. Sheffield & 7.02 & $\begin{array}{l}\text { Hong Kong } \\
\text { Polytechnic U. }\end{array}$ & \\
\hline Boston College & 5.42 & Rutgers U. & 0.93 & Uppsala U. & 4.19 & U. London & 0.02 & $\begin{array}{l}\text { U. } \\
\text { Pennsylvania }\end{array}$ & 3.02 & U. Pennsylvania & 6.45 & $\begin{array}{l}\text { U. } \\
\text { Pennsylvania }\end{array}$ & 4.45 & U. Groningen & 6.96 & U. London & 6.94 \\
\hline U. Giessen & 5.14 & $\begin{array}{l}\text { U. New South } \\
\text { Wales }\end{array}$ & 0.88 & U. Giessen & 4.10 & U. Amsterdam & 0.02 & $\begin{array}{l}\text { Vienna U. E\&B } \\
\text { Administration }\end{array}$ & 2.85 & $\begin{array}{l}\text { U. Texas at } \\
\text { Dallas }\end{array}$ & 6.43 & $\begin{array}{l}\text { Hong Kong U. } \\
\text { S\&T. }\end{array}$ & 4.34 & $\begin{array}{l}\text { U. } \\
\text { Pennsylvania }\end{array}$ & 6.90 & & 6.92 \\
\hline $\begin{array}{l}\text { Georgia State } \\
\text { U. }\end{array}$ & 5.03 & $\begin{array}{l}\text { VU U. } \\
\text { Amsterdam }\end{array}$ & 0.80 & Ohaio State U. & 4.05 & $\begin{array}{l}\text { U. Texas at } \\
\text { Dallas }\end{array}$ & 0.02 & U. Sheffield & 2.85 & Ohio State U. & 5.90 & $\begin{array}{l}\text { U. North } \\
\text { Carolina }\end{array}$ & 4.14 & $\begin{array}{l}\text { U. North } \\
\text { Carolina }\end{array}$ & 6.72 & Indiana U. & 6.59 \\
\hline $\begin{array}{l}\text { Southern New } \\
\text { Hampshire U. }\end{array}$ & 4.55 & U. Amsterdam & 0.79 & U. Maryland & 3.78 & U. Groningen & 0.01 & U. Groningen & 2.62 & $\begin{array}{l}\text { U. North } \\
\text { Carolina }\end{array}$ & 5.56 & $\begin{array}{l}\text { Hong Kong } \\
\text { Polytechnic U. }\end{array}$ & 4.07 & $\begin{array}{l}\text { U. New South } \\
\text { Wales }\end{array}$ & 6.31 & U. Leeds & 6.54 \\
\hline U. Leeds & 4.48 & Uppsala U. & 0.77 & $\begin{array}{l}\text { Hong Kong U. } \\
\text { S\&T. }\end{array}$ & 3.50 & U. Giessen & 0.01 & U. Amsterdam & 2.56 & U. Groningen & 5.38 & U. Groningen & 4.04 & $\begin{array}{l}\text { Hong Kong } \\
\text { Polytechnic U. }\end{array}$ & 6.03 & U. Pennsylvania & 6.38 \\
\hline Ohaio State U. & 4.46 & $\begin{array}{l}\text { Vienna U. E\&B } \\
\text { Administration }\end{array}$ & 0.76 & $\begin{array}{l}\text { Georgia State } \\
\text { U. }\end{array}$ & 3.48 & $\begin{array}{l}\text { U. New South } \\
\text { Wales }\end{array}$ & 0.01 & $\begin{array}{l}\text { U. Texas at } \\
\text { Dallas }\end{array}$ & 2.45 & Uppsala U. & 5.36 & Ohio State U. & 3.98 & $\begin{array}{l}\text { Georgia State } \\
\text { U. }\end{array}$ & 5.72 & U. Giessen & 6.24 \\
\hline $\begin{array}{l}\text { U. North } \\
\text { Carolina at } \\
\text { Greensboro }\end{array}$ & 4.43 & Ohaio State U. & 0.74 & U. Sheffield & 3.48 & $\begin{array}{l}\text { U. Texas at } \\
\text { Austin }\end{array}$ & 0.01 & $\begin{array}{l}\text { Hong Kong } \\
\text { Polytechnic U. }\end{array}$ & 2.35 & $\begin{array}{l}\text { U. New South } \\
\text { Wales }\end{array}$ & 5.29 & Boston College & 3.97 & U. Giessen & 5.70 & INSEAD & 5.98 \\
\hline $\begin{array}{l}\text { U. New South } \\
\text { Wales }\end{array}$ & 4.43 & U. Sheffield & 0.70 & $\begin{array}{l}\text { U. North } \\
\text { Carolina at } \\
\text { Greensboro }\end{array}$ & 3.48 & $\begin{array}{l}\text { Pennsylvania } \\
\text { State U. }\end{array}$ & 0.01 & U. Giessen & 2.34 & U. Sheffield & 4.98 & $\begin{array}{l}\text { U. New South } \\
\text { Wales }\end{array}$ & 3.88 & Boston College & 5.55 & Rutgers U. & \\
\hline U. Sheffield & 4.41 & U. T. Sydney & 0.67 & $\begin{array}{l}\text { U. New South } \\
\text { Wales }\end{array}$ & 3.40 & $\begin{array}{l}\text { Arizona State } \\
\text { U. }\end{array}$ & 0.01 & Uppsala U. & 2.25 & $\begin{array}{l}\text { U. Texas at } \\
\text { Austin }\end{array}$ & 4.75 & $\begin{array}{l}\text { U. the West of } \\
\text { England }\end{array}$ & 3.79 & U. Maryland & 5.52 & U. New South Wales & \\
\hline Indiana U. & 4.34 & $\begin{array}{l}\text { Georgia State } \\
\text { U. }\end{array}$ & 0.64 & $\begin{array}{l}\text { Hong Kong } \\
\text { Polytechnic U. }\end{array}$ & 3.30 & Uppsala U. & 0.01 & $\begin{array}{l}\text { Arizona State } \\
\text { U. }\end{array}$ & 2.25 & $\begin{array}{l}\text { Pennsylvania } \\
\text { State U. }\end{array}$ & 4.67 & U. Maryland & 3.78 & U. Leeds & 5.43 & U. Texas at Dallas & \\
\hline $\begin{array}{l}\text { Hong Kong } \\
\text { Polytechnic U. }\end{array}$ & 4.32 & Boston College & 0.62 & $\begin{array}{l}\text { U. Texas at } \\
\text { Dallas }\end{array}$ & 3.11 & $\begin{array}{l}\text { Chinese U. } \\
\text { Hong Kong }\end{array}$ & 0.01 & $\begin{array}{l}\text { Georgia State } \\
\text { U. }\end{array}$ & 2.16 & Arizona State U. & 4.57 & U. Leeds & 3.70 & $\begin{array}{l}\text { Hong Kong U. } \\
\text { S\&T. }\end{array}$ & 5.31 & U. Manchester & \\
\hline U. Manchester & 4.25 & $\begin{array}{l}\text { National } \\
\text { Chengchi U. }\end{array}$ & 0.60 & U. Amsterdam & 3.09 & $\begin{array}{l}\text { Vienna U. E\&B } \\
\text { Administration }\end{array}$ & 0.01 & $\begin{array}{l}\text { U. Texas at } \\
\text { Austin }\end{array}$ & 2.14 & $\begin{array}{l}\text { Hong Kong } \\
\text { Polytechnic U. }\end{array}$ & 4.55 & $\begin{array}{l}\text { Georgia State } \\
\text { U. }\end{array}$ & 3.67 & U. Amsterdam & 5.24 & Ohaio State U. & 5.02 \\
\hline $\begin{array}{l}\text { National } \\
\text { Chengchi U. }\end{array}$ & 4.16 & $\begin{array}{l}\text { U. the West of } \\
\text { England }\end{array}$ & 0.59 & U. Manchester & 3.08 & $\begin{array}{l}\text { U. North } \\
\text { Carolina at } \\
\text { Greensboro }\end{array}$ & 0.01 & $\begin{array}{l}\text { U. New South } \\
\text { Wales }\end{array}$ & 2.11 & Georgia State U. & 4.50 & U. Giessen & 3.66 & Ohio State U. & 5.22 & Florida State U. & 4.35 \\
\hline
\end{tabular}




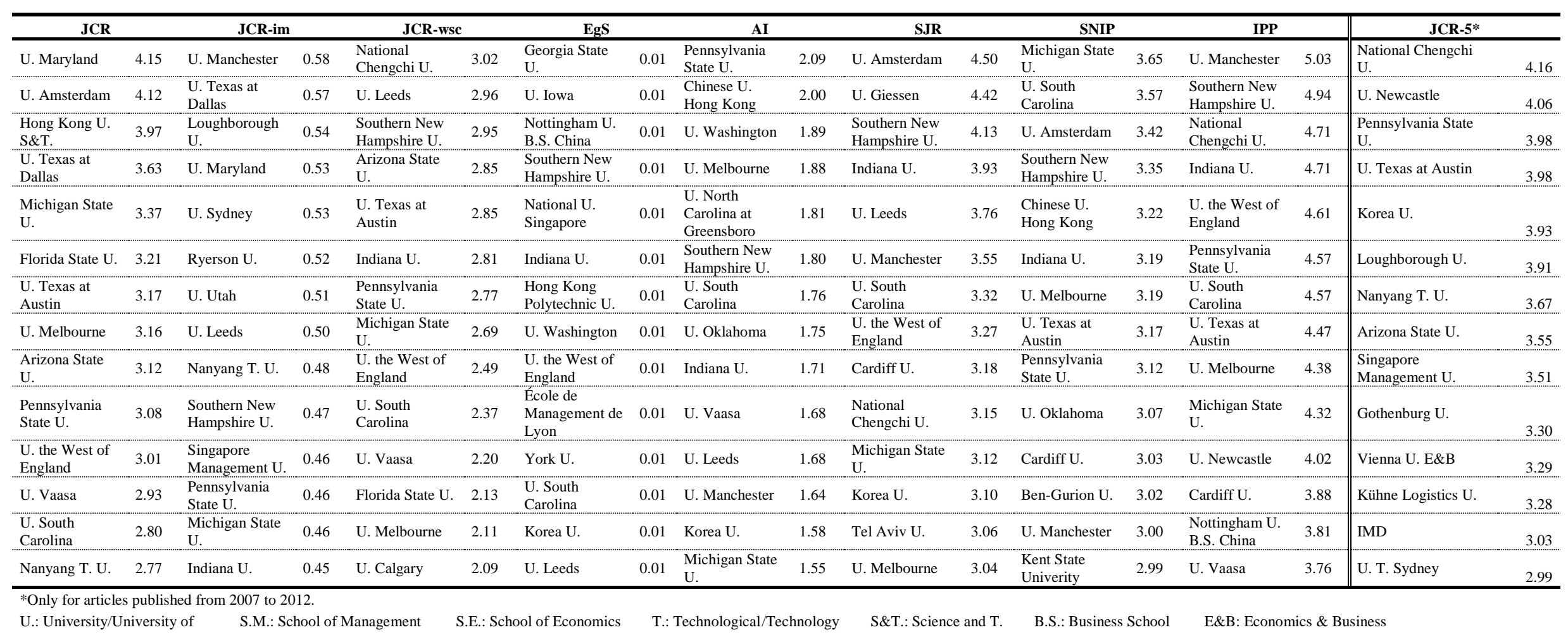


The prominent position of European institutions and the scarce presence of Asia-Pacific institutions must again be acknowledged. As it was the case for prolific institutions, our results are not in line with existing analyses relative to the entire IB field - see, for instance, Treviño et al. (2010). It seems that academic institutions coming from the Asia-Pacific play (or are expected to play) a key role within IB research and agenda, but not in the particular area dealing with the role of NC/CD in IB decisions.

\section{CITATION ANALYSIS}

Understanding what are the key approaches which drive a research field requires observing the influence of the published research, i.e. the most cited works. While the most prolific authors are recognized for producing high quality research, the most influential pieces of research may be authored by less prolific authors (for an example see Pinto et al. (2014). Thus it is important to analyze the citations each paper has received since any citation included in an article reflects some influence on the author's results and/or perspective, posited as an explicit recognition of an intellectual debt (Kochen, 1987). Even assuming the list of reasons for citing a work is extensive, and some negative reasons exist within this list, citations provide a formalized account of the information use and can be taken as a strong indicator of the work's reception by colleagues (Glänzel and Schoepflin, 1999). Therefore we use citation analysis as an objective way to measure the contributions of articles, and has previously been used to assess the influence not only of articles but also individuals, institutions, and journals to a particular field of knowledge see, for instance, Acedo and Casillas (2005), Chandy and Williams, 1994; Li and Tsui (2002), Peng and Zhou (2006), Quer et al. (2007). 


\section{An analysis of the most influential works}

In order to assess the actual impact of the articles gathered in our database, we conducted a citation analysis up to 31 December 2015 using the Scopus database. Citations were not counted

for articles published in journals not included in this database for some particular years ${ }^{12}$, so that we finally measured citation for 256 articles that gathered a total of 9,430 cites $(8,821$ excluding self-citation), an average of more than 36/34 cites per article. Table 6 reports the list of 55 articles with at least 50 cites. Although we have provided at least a three-year period for articles to be cited, it must be acknowledged that this analysis does not do full justice to articles published in most recent years: no article published in 2012 is in this list and only one article published in 2011 is among the most cited ones (Crossland and Hambrick, 2011). In order to take into account this bias we have considered also a relative citation ratio (i.e.: the ratio of citations per year, considering the number of years since the article was published).

\footnotetext{
${ }^{12}$ E.g.: Management International Review (2000-2004).
} 
Table 6. Most cited articles

\begin{tabular}{|c|c|c|c|c|c|c|c|c|c|}
\hline$\underset{E}{*}$ & $\frac{0}{2}$ & $\stackrel{\overline{3}}{\stackrel{0}{3}}$ & 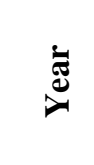 & 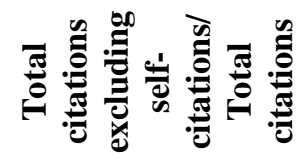 & $\begin{array}{l}\mathscr{\Xi} \\
\simeq\end{array}$ & $\frac{0}{2}$ & 疍 & $\underset{\varpi}{\varpi}$ & 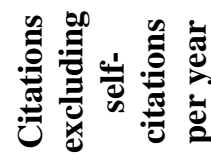 \\
\hline 1 & Johanson \& Vahlne & JIBS & 2009 & $513 / 522$ & 1 & Johanson \&Vahlne & JIBS & 2009 & 85.50 \\
\hline 2 & Tihanyi et al. & JIBS & 2005 & $238 / 245$ & 2 & Tihanyi et al. & JIBS & 2005 & 23.80 \\
\hline 3 & Delios \& Henisz & SMJ & 2003 & $171 / 180$ & 3 & Slangen \& Van Tulder & IBR & 2009 & 22.00 \\
\hline 4 & Brouthers \& Brouthers & JIBS & 2001 & $161 / 165$ & 4 & Barkema \& Drogendijk & JIBS & 2007 & 21.20 \\
\hline 5 & Pothukuchi et al. & JIBS & 2002 & $145 / 146$ & 5 & Berry et al. & JIBS & 2010 & 14.25 \\
\hline 6 & Evans \& Mavondo & JIBS & 2002 & $144 / 147$ & 6 & Crossland \& Hambrick & SMJ & 2011 & 14.22 \\
\hline 7 & Waldman et al. & JIBS & 2006 & $128 / 138$ & 7 & Slangen & JWB & 2006 & 14.00 \\
\hline 8 & Johnson et al. & JIBS & 2006 & $124 / 125$ & 8 & Johnson et al. & JIBS & 2006 & 13.78 \\
\hline 9 & Shimizu et al. & JIM & 2004 & $120 / 127$ & 9 & Shimizu et al. & JIM & 2004 & 12.13 \\
\hline $10-11$ & Berry et al. & JIBS & 2010 & $110 / 114$ & 10 & Zaheer \& Zaheer & JIBS & 2006 & 12.00 \\
\hline $10-11$ & Zhang et al. & JIBS & 2003 & $110 / 118$ & 11 & Stahl et al. & JIBS & 2010 & 11.50 \\
\hline 12 & Skarmeas, et al. & JIBS & 2002 & $108 / 113$ & 12 & Luo & $\mathrm{JM}$ & 2002 & 11.40 \\
\hline 13 & Stahl et al. & JIBS & 2010 & $106 / 113$ & 13 & Takeuchi et al. & AMJ & 2005 & 11.38 \\
\hline 14 & Takeuchi et al. & AMJ & 2005 & $104 / 112$ & 14 & Gong & AMJ & 2003 & 11.17 \\
\hline 15 & Gong & AMJ & 2003 & $103 / 107$ & 15 & Delios \& Henisz & SMJ & 2003 & 11.15 \\
\hline 16 & Larrson \& Lubatkin & HR & 2001 & $100 / 101$ & 16 & Waldman et al. & JIBS & 2006 & 11.08 \\
\hline 17 & Björkman et al. & JIBS & 2007 & $97 / 109$ & 17 & Björkman et al. & JIBS & 2007 & 10.91 \\
\hline 18 & Brannen \& Salk & HR & 2000 & $95 / 102$ & 18 & Drogendijk \& Slangen & IBR & 2006 & 10.71 \\
\hline 19 & Paterson \& Brock & IBR & 2002 & $93 / 94$ & 19 & Mäkelä et al. & JWB & 2010 & 10.67 \\
\hline $20-21$ & Barkema \& Drogendijk & JIBS & 2007 & $91 / 92$ & 20 & Katsikeas et al. & JIBS & 2009 & 10.40 \\
\hline $20-21$ & Makino \& Neupert & JIBS & 2000 & $91 / 93$ & 21 & Sarala \& Vaara & JIBS & 2010 & 10.38 \\
\hline 22 & Luo & JM & 2002 & $88 / 88$ & 22 & Demirbag et al. & MIR & 2008 & 10.33 \\
\hline 23 & Delios \& Henisz & JIBS & 2003 & $87 / 90$ & 23 & Sakarya et al. & IMR & 2007 & 10.20 \\
\hline 24 & Luo & ASQ & 2001 & $86 / 101$ & 24 & Sirmon \& Lane & JIBS & 2004 & 10.17 \\
\hline 25 & Sirmon \& Lane & JIBS & 2004 & $85 / 87$ & 25 & Schwens et al. & JMS & 2011 & 9.67 \\
\hline 26 & Filatotchev et al. & JIBS & 2007 & $83 / 100$ & 26 & Wu et al. & JIBS & 2007 & 9.60 \\
\hline 27 & Drogendijk \& Slangen & IBR & 2006 & $81 / 86$ & 27 & Sousa et al. & JIMk & 2010 & 9.33 \\
\hline 28-30 & Hennart \& Zeng & JIBS & 2002 & $78 / 79$ & 28 & Skarmeas et al. & JIBS & 2002 & 9.29 \\
\hline 28-30 & Manev \& Stevenson & JIBS & 2001 & $78 / 80$ & 29 & Dow \& Ferencikova & IBR & 2010 & 9.20 \\
\hline 28-30 & Zaheer \& Zaheer & JIBS & 2006 & $78 / 80$ & 30 & Brouthers \& Brouthers & JIBS & 2001 & 9.17 \\
\hline 31 & Shenkar et al. & AMR & 2008 & $75 / 79$ & 31-32 & Vaara et al. & JMS & 2012 & 9.00 \\
\hline
\end{tabular}




\begin{tabular}{|c|c|c|c|c|c|c|c|c|c|}
\hline$\underset{\mathscr{E}}{\stackrel{*}{E}}$ & $\frac{0}{e}$ & $\underset{\mathfrak{Z}}{\overline{0}}$ & 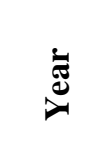 & 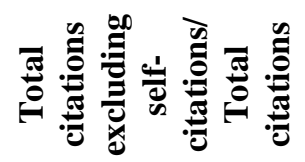 & Ё & $\frac{0}{2}$ & $\underset{\mathfrak{\Xi}}{\overline{0}}$ & $\dot{\Xi}$ & 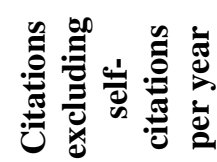 \\
\hline 32-33 & Blomstermo et al. & IMR & 2006 & $72 / 72$ & $31-32$ & Luo & AMJ & 2005 & 9.00 \\
\hline 32-33 & Slangen \& Van Tulder & IBR & 2009 & $72 / 75$ & 33 & Zhang et al. & JIBS & 2003 & 8.67 \\
\hline 34-36 & Katsikeas et al. & JIBS & 2009 & $67 / 72$ & 34 & Pothukuchi et al. & JIBS & 2002 & 8.58 \\
\hline 34-36 & Li et al. & JIBS & 2001 & $67 / 76$ & $35-36$ & Jean et al. & JIMk & 2010 & 8.40 \\
\hline 34-36 & Van Vianen et al. & AMJ & 2004 & $67 / 69$ & $35-36$ & Brock et al. & JIBS & 2008 & 8.40 \\
\hline 37 & Ellis & JIBS & 2008 & $65 / 66$ & 37 & Chen et al. & AMJ & 2010 & 8.31 \\
\hline $38-40$ & Ferner et al. & JWB & 2001 & $64 / 67$ & $38-40$ & Dow \& Larimo & JIMk & 2009 & 8.00 \\
\hline $38-40$ & Luo & AMJ & 2005 & $64 / 70$ & $38-40$ & Makino \& Neupert & JIBS & 2000 & 8.00 \\
\hline $38-40$ & Reus \& Lamont & JIBS & 2009 & $64 / 67$ & $38-40$ & Hutzschenreuter \&Voll & JIBS & 2008 & 8.00 \\
\hline 41 & Ambos \& Ambos & JIM & 2009 & $61 / 64$ & 41 & Chakrabarti et al. & JIBS & 2009 & 7.73 \\
\hline 42 & Slangen & JWB & 2006 & $60 / 61$ & 42 & Stahl \& Caligiuri & JAP & 2005 & 7.60 \\
\hline 43-45 & Evans et al. & IMR & 2000 & $59 / 60$ & 43 & Delios \& Henisz & JIBS & 2003 & 7.38 \\
\hline $43-45$ & Tong \& Reuer & JIBS & 2007 & $59 / 64$ & 44 & Morschett et al. & JIM & 2010 & 7.25 \\
\hline $43-45$ & Uhlenbruc & JIBS & 2004 & $59 / 59$ & 45 & Reus \& Lamont & JIBS & 2009 & 7.15 \\
\hline $46-48$ & Arora \& Fosfuri & JIBS & 2000 & $58 / 59$ & 46 & Evans \& Mavondo & JIBS & 2002 & 7.14 \\
\hline $46-48$ & Chakrabarti et al. & JIBS & 2009 & $58 / 60$ & 47 & Arora \& Fosfuri & JIBS & 2000 & 7.00 \\
\hline $46-48$ & Vaara et al. & HR & 2003 & $58 / 63$ & 48 & Demirbag et al. & JWB & 2007 & 6.86 \\
\hline 49 & Chen et al. & AMJ & 2010 & $57 / 57$ & 49 & Campbell et al. & JIBS & 2012 & 6.77 \\
\hline $50-51$ & Crossland \& Hambrick & SMJ & 2011 & $56 / 59$ & $\mathbf{5 0}$ & Hartmann et al. & JWB & 2010 & 6.67 \\
\hline $50-51$ & Griffith et al. & JIBS & 2000 & $56 / 64$ & 51 & Chari \& Chang & JIBS & 2009 & 6.57 \\
\hline $52-53$ & Dow \& Larimo & JIMk & 2009 & $54 / 57$ & 52 & Aybar\& Ficici & JIBS & 2009 & 6.40 \\
\hline $52-53$ & Schlegelmilch \& Chini & IBR & 2003 & $54 / 54$ & 53 & Shenkar et al. & AMR & 2008 & 6.33 \\
\hline 54 & Stahl \& Caligiuri & JAP & 2005 & $52 / 53$ & 54 & Ambos \& Ambos & JIM & 2009 & 6.14 \\
\hline 55 & Morschett et al. & JIM & 2010 & $51 / 53$ & 55 & Van Vianen et al. & AMJ & 2004 & 6.13 \\
\hline
\end{tabular}

*Ranked by total number of citation, excluded self-citations. 
Observing Table 6 we may identify some particularly recent articles are among the most cited ones in relative terms. The subjects of the most recent papers are rather mixed, but we may identify a growing interest in the institutional approach. For instance, Crossland and Hambrick's (2011) study on the influence of formal and informal national institutions on managerial discretion of CEOs; Schwens et al.'s (2011) study of the influence of formal and informal institutions in the choice of entry mode by small and medium size firms; and Berry et al.'s (2010) proposal of a multidimensional measure of institutional distance. Other recent articles which are highly influential include papers which focus on psychic distance (e.g. Dow and Ferencikova, 2010; Dow and Larimo, 2009) which is includes national cultural differences but also other national-level differences (Johanson and Vahlne, 1977). More recently published articles which are gaining quick influence, specifically Campbell et al. (2012) — an analysis of the influence of CD in multinationals corporate social responsibility activities — and Vaara et al. (2012) — a study on the impact of organizational and national cultural differences on social conflict and knowledge transfer in international acquisitions, address other host-country dimensions which are posited to influence firms' operations abroad. These articles are not among the most cited ones in absolute terms; however, their high relative citation ratio points to an expected high influence in the near future. Therefore we may identify a growing influence of research addressing the multiple challenges of international business environment which go beyond national cultural differences.

Although one of the most common criticism placed on citation analysis is the unequal frequency of citation of conceptual and empirical articles (Chandy and Williams. 1994; Harzing, 2002), the percentage of conceptual articles within this selected list - $18 \%$ - is not too far from the percentage of this kind of articles in the database - 12\%, as shown in LVG (2015). However, it 
is worth nothing that this percentage raises to $27 \%$ when focusing on the top-10 most cited articles. Among these it is the most cited article within the database: the piece of research by Johanson and Vahlne (2009) aimed at revisiting the Uppsala internationalization model in the light of new business practices and recent theoretical advances (over 500 citations in just 6 years). Johnson et al. (2006) - an article that develops a model of cross cultural competences to be applied in international business and international human resource management - and Shimizu et al. (2004) — an analysis of cross border mergers and acquisitions as entry mode in foreign markets, dynamic learning process from a foreign culture, and value creating strategyare the other two conceptual articles within the top-10 list.

One third of the top-10 most cited articles deal with foreign direct investments: the meta-analysis by Tihanyi et al. (2005) focused on the impact of $\mathrm{CD}$ on entry mode choice and performance, the study by Brouthers and Brouthers (2001) centered on the role played by the interaction between CD and host country risk on the choice between wholly owned subsidiaries and joint ventures, and Delios and Henisz's (2003b) study aimed at extending the sequential model of internationalization by incorporating the policy environment as a source of uncertainty to a firm whose relevance can be similar to that of cultural and social environment. There is only one work related to international alliances within this top-10 list: Pothukuchi et al.'s (2002) study centered on the analysis of national and organizational culture differences on international joint ventures performance. The articles by Evans and Mavondo (2002) — an exploration of a comprehensive measure of psychic distance and its influence in organizational performance-, Waldman et al. (2006) — an analysis of the cultural and leadership variables associated with corporate social responsibility values-, Berry et al. (2010) - a proposal of a multidimensional measure of cross national distance and an empirical exploration of its influence in foreign expansion choices-, 
and Zhang et al. (2003) — a study of the influence of cultural an country differences on relational norms and trust-building activities between cuyers and sellers - complete the top-10 list of most cited articles.

\section{An analysis of the most influential authors and institutions}

A total of 120 authors and 80 academic institutions ${ }^{13}$ are involved in the most cited articles gathered in Table 6. From this list we selected the top 25 -most-cited authors by estimating cumulative cites based on these 55 articles (actually 28 authors, as 4 different scholars share the $25^{\text {th }}$ position; see Table 7).

Table 7. Top authors based on cumulative citations

\begin{tabular}{clcclc}
\hline Rank & \multicolumn{1}{c}{ Authors } & $\begin{array}{c}\text { Total citations } \\
\text { excluding self } \\
\text { citations }\end{array}$ & Rank & \multicolumn{1}{c}{ Authors } & $\begin{array}{c}\text { Total citations } \\
\text { excluding self } \\
\text { citations }\end{array}$ \\
\hline $\mathbf{1 - 2}$ & Johanson, Jan & 513 & $\mathbf{1 5}$ & Drogendijk, Rian & 172 \\
$\mathbf{1 - 2}$ & Vahlne, Jan-Erik & 513 & $\mathbf{1 6}$ & Schlegelmilch, Bodo B. & 162 \\
$\mathbf{3}$ & Luo, Yadong & 313 & $\mathbf{1 7 - 1 8}$ & Brouthers, Keith D. & 161 \\
$\mathbf{4}$ & Griffith, David A. & 294 & $\mathbf{1 7 - 1 8}$ & Brouthers, Lance & 161 \\
$\mathbf{5 - 6}$ & Delios, Andrew & 258 & $\mathbf{1 9}$ & Vaara, Eero & 155 \\
$\mathbf{5 - 6}$ & Henisz, Witold J. & 258 & $\mathbf{2 0 - 2 4}$ & Chen, Chao C. & 145 \\
$\mathbf{7}$ & Stahl, Gunter & 255 & $\mathbf{2 0 - 2 4}$ & Choi, Jaepil & 145 \\
$\mathbf{8 - 9}$ & Russel, Craig J, & 238 & $\mathbf{2 0 - 2 4}$ & Damanpour, Fariborz & 145 \\
$\mathbf{8 - 9}$ & Tihanyi, Laszlo & 238 & $\mathbf{2 0 - 2 4}$ & Park, Seung Ho & 145 \\
$\mathbf{1 0}$ & Slangen, Arjen & 213 & $\mathbf{2 0 - 2 4}$ & Pothukuchi, Vijay & 145 \\
$\mathbf{1 1 - 1 2}$ & Evans, Jody & 203 & $\mathbf{2 5 - 2 8}$ & de Luque, Mary Sully & 128 \\
$\mathbf{1 1 - 1 2}$ & Mavondo, Felix T. & 203 & $\mathbf{2 5 - 2 8}$ & House, Robert & 128 \\
$\mathbf{1 3 - 1 4}$ & Katsikeas, Constantine S. & 175 & $\mathbf{2 5 - 2 8}$ & Waldman, David A. & 128 \\
$\mathbf{1 3 - 1 4}$ & Skarmeas, Dionisis & 175 & $\mathbf{2 5 - 2 8}$ & Washbur, Nathan & 128 \\
\hline
\end{tabular}

As shown in Li and Tsui (2002), this is a conservative estimate of the impact of these authors' work, as we did not include in this cumulative counter other articles they published that did not survive the selected cutoff of 50 citations. Table 7 shows that Johanson and Vahlne are the only authors with over 500 citations of their work, Luo has more than 300 citations, and 9 different

\footnotetext{
13 The article by Waldman et al. (2006) has 4 main authors and 37 additional collaborators. Only the main authors and their respective institutions have been included in this ranking.
} 
authors gather more than 200 citations (Griffith, Delios, Henisz, Stahl, Russel, Tihanyi, Slangen, Evans, and Mavondo). It is noteworthy to point that Johanson and Vahlne have only one article (co-authored by both of them) within this selected list, so that their clear prominent position is exclusively due to this particular piece of research. Following $\mathrm{Xu}$ et al. (2008), we have gathered information about these top-authors' current institutional affiliation of (Table 8). 
Table 8. Institutions currently hosting the most cited authors

\begin{tabular}{lc}
\hline \multicolumn{1}{c}{ Institution } & Country \\
\hline Aalto U. School of Business & Finland \\
Arizona State U. & United States \\
Athenes U. Economics & Greece \\
China Europe International Business School & China \\
Erasmus U. Rotterdam & Netherlands \\
Goteborgs U. & Sweden \\
Kennesaw State U. & United States \\
Lehigh U. & United States \\
Monash U. & Australia \\
National U. Singapore & Singapore \\
Rutgers, The State U. New Jersey & United States \\
Sungkyunkwan U. & South Korea \\
Texas A\&M U. & United States \\
U. Groningen & Netherlands \\
U. Leeds & United Kingdom \\
U. London & United Kingdom \\
U. Melbourne & Australia \\
U. Miami & United States \\
U. Oklahoma & United States \\
U. Pennsylvania & United States \\
Uppsala U. & Sweden \\
Utah State U. & United States \\
Vienna U. Economics and Business Administration & Austria \\
\hline U.: University of/University &
\end{tabular}

It is to be expected that the institutions where these scholars are currently working hold considerable influence on the research field in the near future. As we can see in the table, it may be expected that some institutions that are not currently in top positions within the volume/productivity/citation rankings emerge and/or improve their positioning. We may identify European (e.g.: Aalto University-Finland, Athens University of Economics and Business-Greece, University of Groningen-Netherlands, Göteborgs University-Sweden), US (e.g.: Kennesaw State University, Lehigh University, Utah State University) and Asia-Pacific institutions (e.g.: China Europe International Business School-China, National University of Singapore-Singapore, Sungkyunkwan University-South Korea) which may increase their influence. The geographic 
pattern shows a balanced distribution among European and North American institutions, as well as a slight increase in the weight of academic institutions coming from the Asia-Pacific region.

\section{SCIENTIFIC COLLABORATION}

The study of collaboration trends informs about the relevance of research teams and networks, as well as about the relevance of different kinds of collaborative research (intramural, national, and international). Less than $20 \%$ of the 265 articles are single-authored. This result points to the relevance of team-projects and networks of scholars that facilitate access to resources (i.e.: expertise, new skills, equipment, funds), increase efficiency, allow tackling "bigger" problems or challenges, and/or improve scholars' prestige and visibility (Beaver, 2001). This particularly high rate of co-authored articles is consistent with the publication trends already pointed by Inkpen and Beamish (1994) and Floyd et al. (1994) in IB and Management journals respectively. As shown in both studies, the publication pattern has consistently evolved towards collaborative research. Furthermore, this tendency is not exclusive of the business and management fields, as scientific collaboration -as measured by means of co-authorship patterns- has considerably increased during the last decades at all levels of aggregation in a wide range of scientific fields (Glänzel, 2001).

Our analysis points to a particularly high number of multi-authored articles (more than $42 \%$ of the articles are co-authored by 3 or more researchers). This publication pattern points to the relevance of participating in large research teams or networks in order to achieve publishing in the top-tier journals analyzed in this study. This pattern can be related to the low rate of exclusive intramural collaborative research (collaboration among authors working at the same academic institution), as less than $20 \%$ of co-authored articles reflect exclusive intramural co-authorship. 
Conversely, up to $41.5 \%$ of total articles and $50 \%$ of co-authored articles reflect international collaboration (i.e.: the authors' institutional affiliation includes more than one country). Both the increase of the density of the networks and the intensification of international collaboration are patterns also identified in different studies related to other research fields (Glänzel, 2001), but scarcely explored in the IB and Management fields. A cross-tab analysis of the international collaboration ratio in the articles gathered in the database and the year/journal International Collaboration ratios provided by Scopus for the selected list of journals searched in this study shows that the presence of international collaboration within this research area is higher than average.

In order to analyze the potential influence of international collaboration on scholars' productivity as measured by journal impact factors, we divided the database in two sub-samples based on presence/absence of international collaboration. We then performed a mean difference analysis for each of the 9 journal metrics mentioned before. No statistical differences have been found. Therefore, our study does not show a positive effect of international collaboration on achieving journals with higher impact factors. Conversely, international collaboration seems to play a positive influence on articles actual impact as measured by number of citations: the percentage of articles showing an international co-authorship pattern raises to 52\% among the most cited articles.

\section{ANALYSIS OF THE KNOWLEDGE BASE}

The analysis of the knowledge base studies the bibliographic references included in the set of researched articles in order to identify not only the most relevant individual references, but also clusters of closely related documents, as well as potential interrelations among them. 
As a first step, it examines the bibliographic references cited in research documents as an indicator of the sources of information used and the approaches connected or integrated in their work (Garfield, 1979; Kochen, 1987; Smith, 1981). Therefore, this analysis allows identifying the works that have exerted the highest influence on the perspectives, development, and results of the articles being studied. It bases on the same assumption as the citation analysis - a work cited more often is more influential to a given field-, but as a reverse process as it analyzes the works that have been cited by the articles included in the database.

As a second step and in order to examine potential interrelations, we also computed the number of times that two documents appear jointly cited (i.e.: co-citation analysis). This allows identifying clusters of related documents and their potential interrelation. Clusters are formed by selecting all papers that can be linked together by a specified joint-use threshold. This analysis explores the research fronts of the field under study; that is, the groups of highly cited and interrelated papers referred to as core papers in the field. It is worth noting that although the works within a specific research front address the same questions, they do not necessarily have to share the same perspective or agree with each other. This is a technique used widely to identify the structure of knowledge in different fields of study — see Acedo and Casillas (2005) and Dagnino et al. (2015) for exhaustive reviews.

To perform these analyses we retrieved the relevant meta-information (Zupic and Cater, 2014) from the 265 articles in our sample, including the 18,593 unique references (averaging 70.2 per article). We examined all the references to consolidate any duplicate versions, misspellings of the authors' names or inconsistencies of the volumes or page numbers. We also consolidated every book reference to account for different editions of the same work. We then followed the 
procedure put forward by Ferreira et al. (2014a) to identify the most influential works within the field as well as how they interconnect to support the extant literature.

Table 10 presents the 40 most frequently used references ${ }^{14}$. The table shows that almost $80 \%$ of these references are articles published in academic journals (22\% of them are books), as well as the prevalent role of the Journal of International Business Studies as main source of referenced articles - more than $40 \%$ of these selected references come from this journal. Two management journals - the Strategic Management Journal and the Academy of Management Journal- also play a particularly relevant role, as they gather jointly over $20 \%$ of these references.

\footnotetext{
14 Three of these articles (i.e.: Barkema et al., 1996; Kim and Hwang 1992; and Parkhe, 1991) and 11 different authors gathered in this list are also included in the ranking of most cited an influential articles/authors in global strategy research published during the 90 s (Peng and Zhou, 2006).
} 
Table 10. Most cited works by the articles included in the database

\begin{tabular}{|c|c|c|c|c|c|}
\hline Rank & Work & Citations & Rank & Work & Citations \\
\hline 1 & Hofstede (1980) & 219 & 21 & O’Grady \& Lane (1996) & 29 \\
\hline 2 & Kogut \& Singh (1988) & 127 & $22-25$ & Barkema et al. (1997) & 26 \\
\hline 3 & Shenkar (2001) & 76 & $22-25$ & Trompenaars \& Hampden-Turner (1993) & 26 \\
\hline 4 & Johanson \& Vahlne (1977) & 57 & $22-25$ & Brouthers \& Brouthers (2001) & 26 \\
\hline 5 & Hofstede (1991) & 51 & $22-25$ & Johanson \& Wiedersheim-Paul (1975) & 26 \\
\hline 6 & Barkema et al. (1996) & 48 & 26-27 & Parkhe (1991) & 25 \\
\hline 7 & Gatignon \& Anderson (1988) & 43 & 26-27 & Ronen \& Shenkar (1985) & 25 \\
\hline 8-9 & Morosini et al. (1998) & 38 & 28-32 & Armstrong \& Overton (1977) & 24 \\
\hline 8-9 & House et al. (2004) & 38 & 28-32 & Hall (1976) & 24 \\
\hline $10-13$ & Hair et al. (1979) & 36 & 28-32 & Hennart (1988) & 24 \\
\hline 10-13 & Kim \& Hwang (1992) & 36 & 28-32 & Brouthers (2002) & 24 \\
\hline $10-13$ & Bartlett \& Ghoshal (1989) & 36 & 28-32 & Kostova (1999) & 24 \\
\hline $10-13$ & Park \& Ungson (1997) & 36 & 33 & Cohen \& Levinthal (1990) & 23 \\
\hline 14 & Erramilli \& Rao (1993) & 33 & 34-35 & Chang \& Rosenzweig (2001) & 22 \\
\hline $15-16$ & Williamson (1985) & 32 & 35-35 & Aulakh et al. (1996) & 22 \\
\hline $15-16$ & Hennart \& Larimo (1998) & 32 & $36-40$ & Zaheer (1995) & 21 \\
\hline 17 & Barkema \& Vermeulen (1997) & 31 & $36-40$ & Buckley \& Casson (1976) & 21 \\
\hline 18-20 & Barkema \& Vermeulen (1998) & 30 & $36-40$ & Weber et al. (1996) & 21 \\
\hline $18-20$ & Anderson \& Gatignon (1986) & 30 & $36-40$ & Kogut (1988) & 21 \\
\hline 18-20 & Morgan \& Hunt (1994) & 30 & $36-40$ & Hennart \& Reddy (1997) & 21 \\
\hline
\end{tabular}

Source: Authors computations using ISI Web of Knowledge data. 
The most relevant and influential work is Hofstede's (1980) book in which four different dimensions of national culture are introduced, analyzed, and measured for a set of countries, consistent with previous research which have suggested the influence of Hofstede in the last decades (e.g. Chandy and Willims, 1994; Ferreira et al. 2014b; Pinto et al., 2014) The article by Kogut and Singh (1988) is second in the ranking. This is a piece of research focused on the choice of entry mode in foreign direct investment processes, offering an explanation based on a transaction costs theory approach (Ferreira et al., 2014c); however, its prominent position is due to the introduction of an index that integrates in just one unique measurement the above referred four cultural dimensions in order to measure the cultural distance between two countries. In turn, Shenkar's (2001) article is a critical review of this particular CD construct "outlining its hidden assumptions and challenging its theoretical and methodological properties" (Shenkar; 2001, 519). The review analyzes this construct's shortcomings and limitations, as well as their impact on entry mode research. Therefore, the 3 most frequently referenced articles are clearly methodological and focused on the way in which existing cultural distance among countries is (or should be) measured. Hall (1976), Hofstede (1991), House et al. (2004), Ronen and Shenkar (1985), and Trompenaars and Hampden-Turner (1993); are other highly-cited research pieces that also center their attention on this subject.

The article by Johanson and Vahlne (1977) developing the Uppsala internationalization model a gradual or sequential international growth model based on the role played by cumulative knowledge and accrued experience - is the first conceptual article included in the list. It is noteworthy to remember that the "updated" version of this model (Johanson and Vahlne, 2009) is the most cited article included in our dataset. The study of alternative entry mode strategies and 
the analysis of the different factors conditioning the choice among them gather a particularly high number of articles included in this list ${ }^{15}$. Of course, national cultural traits and cultural differences between the home and the target countries of the internationalization process are conditioning factors included in all these studies (e.g.: Barkema et al., 1996; Gatignon and Anderson, 1988; Kim and Hwang, 1992; Erramilli and Rao, 1993). Most of them focus on the choice between alternative entry modes related to foreign direct investment processes (i.e.: choice between wholly-owned subsidiaries and joint ventures or between new start-ups and acquisitions). A second group of articles related to entry mode strategies focuses on the influence of $\mathrm{NC} / \mathrm{CD}$ on the performance and evolution of different entry modes (e.g.: joint ventures, acquisitions) —Barkema and Vermeulen (1997), Morosini et al. (1998), Park and Ungson (1997), Parkhe (1991), among them. The influence of NC/CD on multinationals organization design (e.g.: Barttlet and Goshal, 1989) or on knowledge transfer and absorptive capacity (e.g.: Cohen and Levinthal, 1990; Kostova, 1999) are also among the subjects addressed to by some of these top-referenced articles.

Examining the joint use of references allowed us to understand the interconnectedness of these works and depict the intellectual structure of the field (Ferreira et al., 2014a; Zupic and Cater, 2014). Two works used in the same article suggest some kind of connection between them (White and McCain, 1990). We selected the 40 most cited works in our sample and constructed a co-occurrence matrix to plot a network (Shafique, 2013). Figure 1 presents the co-occurrence network from which we infer the most relevant works and the relation among them. The influence of the reference is depicted both by the position in the network (central positions are

\footnotetext{
${ }^{15}$ The analysis of entry mode strategies is also a major theme addressed by most cited articles in IB research between 1996 and 2006 (Griffith et al., 2008).
} 
more influential) and the size of the node (a larger circle means a more important work), while relations between nodes are depicted by a line; the thicker the line, the stronger the relation - i.e.: the more often two works are used together.

Figure 1. Co-citation network

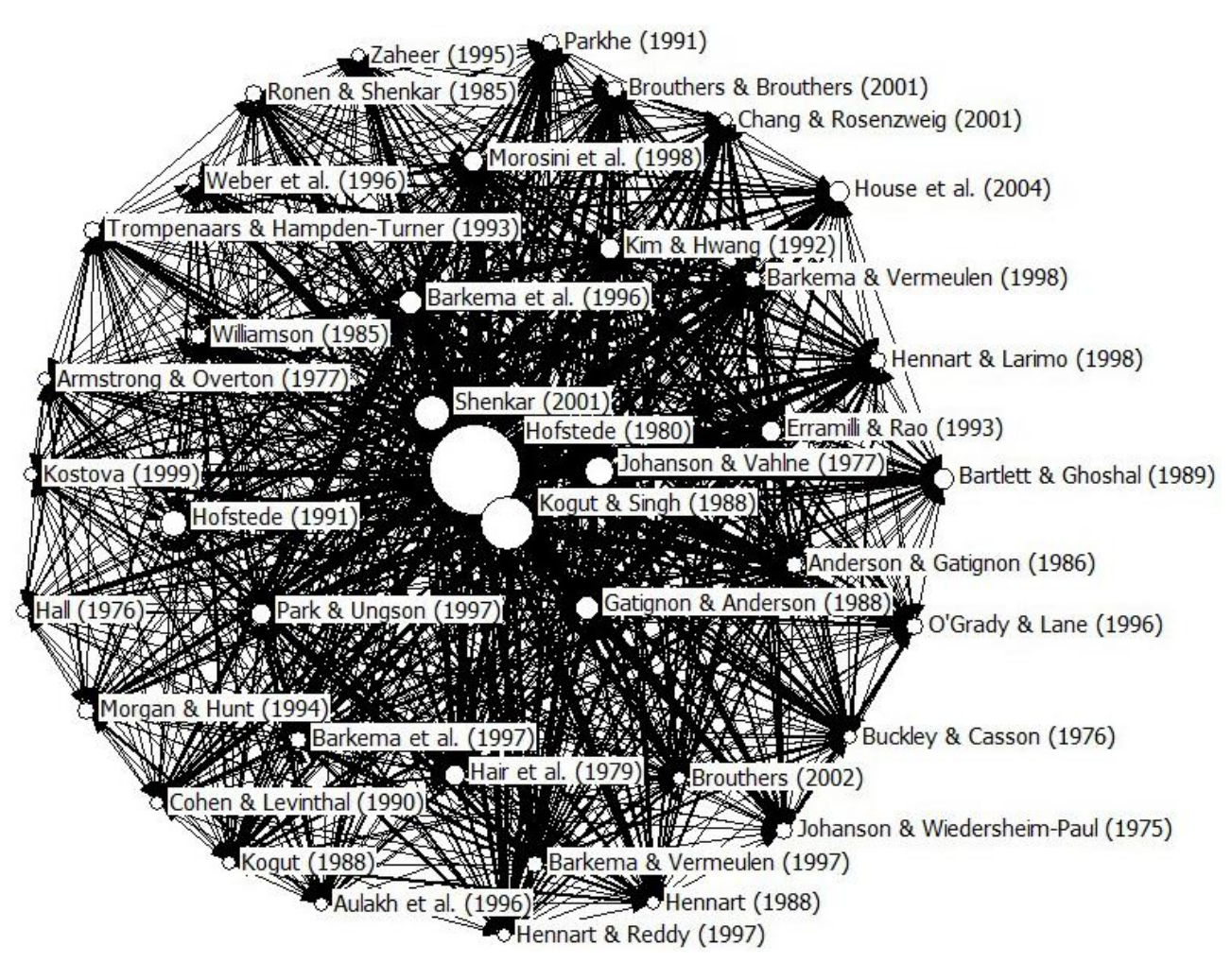

Source: Authors computations using ISI Web of Knowledge data. Network plotted with Ucinet.

Observing the co-citation network we identify a cluster of highly interconnected works at the core dealing with cultural distance measurement (Hofstede, 1980; Kogut and Singh, 1988; Shenkar, 2001). The 3 most frequently cited papers are strongly interconnected, supporting the idea already placed in LVG (2015): regardless of its limitations and shortcomings (Shenkar, 2001), the index developed by Kogut and Singh (1988) to integrate the national cultural dimensions measured by Hofstede (1980) remains as the most frequently used measurement of 
CD within IB literature. Furthermore, the pivotal position of this model is corroborated by the thick lines (i.e.: strong relations) that connect these articles with the different works putting forward alternative cultural models/measurements located in peripheral positions (Hall, 1976; Trompenaars and Hampden-Turner, 1993; House et al., 2004). This evidence suggests that these models/measurements have been used as an alternative to the "standard" or "traditional" measurement most widely accepted within the IB field and that their selection and use requires some kind of justification, comparison with, and/or reference to the "traditional" model.

On a second layer we find a number of works on international strategy issues such as internationalization path (Johanson and Vahlne, 1977) and entry mode choice and/or performance (Barkema et al., 1996, Erramilli and Rao, 1993; Gatignon and Anderson, 1988; Park and Ungson, 1997) that are tightly connected to the core works. This evidence points to a particularly high use of the above referred traditional measurement of $\mathrm{CD}$ in the articles dealing with these issues. Conversely, the above mentioned articles dealing with organizational design, knowledge transfer, and absorptive capacity do not rely so heavily on this model. The articles that base on a particular theoretical approach - e.g.; the analysis related to entry modes based on Transaction Cost Economics by Hennart (1988). Hennart and Larimo (1998), and Hennart and Reddy (1997) — and conceptual pieces of research — e.g.: Buckley and Casson (1976), Zahher (1995)— are located in peripheral positions in the network.

\section{DISCUSSION}

This piece of research is a comprehensive quantitative literature review focused on the relevance of national culture in International Business research. It has been developed as a complement to the qualitative literature review recently published in the International Journal of Management 
Reviews by LVG (2015). LVG's article presents a map of the field and explores its research agenda following a subjective approach based on the authors' interpretation. However, to fully understand the intellectual structure of a field of research both qualitative/subjective and quantitative/objective analyses are needed, as they complement each other (Acedo and Casillas, 2005). Therefore, this quantitative review relies on the same list of 265 articles published in top IB and Management journals previously selected by LVG (2015).

A wide set of bibliometric techniques and indicators (i.e.: absolute and adjusted counting, journals performance, citation analysis, indicators of collaborative research, and analysis of the knowledge base) have been used in order to provide information about institutions and scholars' research productivity and performance, their current and potential influence in the field, and the collaborative patterns they have followed. The article also explores the authors/works that have exerted the greatest influence on the researched body of literature, interconnections among them, and existing research fronts. In short, this study sheds light on the source and magnitude of the scholarly and institutional influence in this area or sub-field within IB.

Our results and rankings must be interpreted cautiously and always keeping in mind that they are contingent to the selected time period and searched journals. Anyhow, it is possible to derive some trends and conclusions. The first conclusion is that in order to have a comprehensive understanding of the ranking of academic institutions and academicians, different measures must be combined. It is clear that a high number of publications in top academic journals (as those searched in this study) clearly indicates that a scholar/institution has been successful in generating high visibility output considered as validated knowledge (Podsakoff et al., 2005; Ramos-Rodríguez and Ruíz-Navarro, 2004). However, this number does not provide information about this output's potential/actual impact. Ranking scholars or institutions by using the number 
of publications in an isolated way hides the implicit assumption that all contributions have equal impact. As pointed in Peng and Zhou (2006), although all published journal articles can be argued to be high quality research which makes a contribution, their impact is not likely to be equal.

Using journals metrics (impact factors and influence scores) is an initial option to overcome this shortcoming (Treviño et al., 2010), as they provide a first approach to the publishing profile of authors and institutions. As a wide range of metrics exist, each of them favoring a particular issue (raw impact, weighted impact, immediacy, etc.), the use of a single metric does not provide a comprehensive oversaw of research potential impact. Just on the contrary, different metrics based on diverse data sources and different methodologies must be considered. Therefore, 9 different metrics have been used in this article in order to measure institutions and authors' productivity. Even when considering multiple metrics, ranking authors and institutions based only on journals' performance may derive in a bias, as these metrics relate to "an average article" published in a specific journal/year.

A citation analysis allows measuring the actual impact of a particular article. A citation to a work means that it has been used and recognized as relevant by the citing scholar and that the cited work is somehow related in content to the citing one (Smith, 1981). Therefore, it must be emphasized that citations measure influence (Starbuck, 1994). Regardless of its objective nature, biases are still likely —older publications getting, on average, a higher number of citations, potential citation based on legitimacy issues, no differentiation between positive and negative citation, miss-citation, or some type of articles (i.e: conceptual, reviews) generally receiving more 
cites (Harzing, 2002; Chandy and Williams; 1994; Glänzel and Schoepflin, 1999; Mizruchi and Fein, 1999; Peng and Zhou, 2006).

As each measure has advantages, but also shortcomings, considering a wide spectrum of measures becomes essential in order to provide a reliable overview of the field. This wide range of measures may collectively help form a fairly accurate assessment of the role authors, journals, and institutions play in contributing to the research field. Actually, our analysis relative to the role played by individual academicians reveals that volume (number of publications), productivity (publications impact based on journals' performance), and influence (number of citations) do not always overlap. In summary, although one may intuitively expect that more prolific authors are also highly productive and cited, this is not necessary the case - our findings relative to this point are consistent with those achieved by Peng and Zhou (2006) in their analysis relative to global strategy literature. In addition, all these measures must be complemented by an analysis of collaborative research patterns, in order to understand the role of individual scholars and research teams, as well as to explore existing interactions among academic and non-academic institutions and the relevance of intramural and extramural collaboration.

The analysis relative to institutions shows similar results relative to a low correlation among most prolific, most productive, and most influential (cited) ones. Our results point to a particularly wide international scope consistent with that already shown in studies regarding the entire IB field (Lahiri and Kumar, 2012; Treviño et al., 2010; Xu et al., 2008). However, a clear differentiated feature of the research within this particular subfield is the prevalent role of European academic institutions over North-American and Asia-Pacific ones. A more balanced 
influence of European and US institutions is expected in the near future, as shown by the analysis of the institutions currently hosting the most influential authors within the field.

Collaborative research and specifically international collaborative research plays a crucial role in this body of literature, as our results show a high number of articles co-authored by large and cross-national teams. It seems that international collaborative research does not influence scholars' performance in terms of achieving journals with higher impact or influence, although it has some influence on articles impact (citation). Collaborative patterns are limited to collaboration ties and networks among academic institutions, as active collaboration between academic institutions and firms or governmental agencies is extremely scarce.

The analysis of the knowledge base shows that methodological issues, specifically those related to the way in which cultural dimensions and cultural distances between countries are measured, remain as a key issue in this field. Furthermore, the co-occurrence analysis points to a particularly wide body of articles using a model/measurement of cultural distance (i.e.: Hofstede's model of cultural dimensions and the index by Kogut and Singh) whose shortcomings and limitations are known in advance by the authors using them (Shenkar, 2001). Their use seems to rely (at least partially) in legitimacy issues (i.e.: former studies using the same measurement that is somehow accepted as an standard within this body of research) and easiness of use, as well as in the lack of an accepted clear alternative.

\section{Limitations and future directions}

Although the present study provides important insights into the state of scholarship in the IB/NC area, it is important to note several limitations. 
We have relied on an already existing list of selected articles dealing with our intended issue. Although this decision has allowed us to complement LVG's (2015) qualitative study, it implies falling into the same shortcomings related to the selection of journals (only top tier academic IB and Management journals) and type of documents (only full-length articles).

As said before, results are contingent to the selected time period. Additionally, past productivity/influence is not necessary and indicator of future one. As stated in Xu et al. (2008), the productivity of authors varies depending on their personal and professional cycles, editorial roles, and so on. In addition, when dealing with institutions, mobility in academia must be taken into account. Although we have considered both the institution hosting the authors at the moment the articles were published and the institutions currently hosting these authors, there is no guarantee that they will keep in the latter. Furthermore, as pointed by Stahl et al. (1988) and Trieschmann et al. (2000) institutional productivity can be related to faculty size, the proportion of full professors, the existence of doctoral programs, and editorships.

An extension of this work using more sophisticated citation methodologies (e.g.: second generation cites, indirect self-citation) — see, for instance, Tahai and Meyer (1999)— could be an interesting issue within the research agenda. Furthermore, as pointed by Peng and Zhou (2006), a meaningful measure of an article's influence would be its impact on the profession rather than on researchers. Although this arises as an exciting challenge, it requires a data source quite different from the one used in this research. 


\section{References}

Acedo, F.J. and Casillas, J.C. (2005). Current paradigms in the international management field: An author co-citation analysis. International Business Review, 14, pp. 619-639.

Ambos, T.C. and Ambos, B. (2009). The impact of distance on knowledge transfer effectiveness in multinational corporations. Journal of International Management, 15, pp. 1-14.

Anderson, E. and Gatignon, H. (1986). Modes of foreign entry: A transaction cost analysis and propositions. Journal of International Business Studies, 17 (3), pp. 1-26.

Armstrong, J.S. and Overton, T.S. (1977). Estimating nonresponse bias in mail surveys. Journal of Marketing Research, 14 (3), pp. 396-402.

Arora, A. and Fosfuri, A. (2000). Wholly owned subsidiary versus technology licensing in the worldwide chemical industry. Journal of International Business Studies, 31 (4), pp. 555-572.

Aulakh, P.S., Kotabe, M. and Sahay, A. (1996). Trust and performance in cross-border marketing partnerships: A behavioral approach. Journal of International Business Studies, 27 (5), pp. 1005-1032.

Aybar, B. and Ficici, A. (2009). Cross-border acquisitions and firm value: An analysis of emerging-market multinationals. Journal of International Business Studies, 40, pp. 1317-1338.

Barkema, H.G. and Drogendijk, R. (2007). Internationalising in small, incremental or larger steps? Journal of International Business Studies, 38 (7), pp. 1132-1148.

Barkema, H.G. and Vermeulen, F. (1997). What differences in the cultural backgrounds of partners are detrimental for international joint ventures?. Journal of International Business Studies, 28 (4), pp. 845-864.

Barkema, H.G. and Vermeulen, F. (1998). International expansion through start-up or acquisition: A learning perspective. Academy of Management Journal, 41 (1), pp. 7-26.

Barkema, H.G., Bell, J.H. and Pennings, J.M. (1996). Foreign entry, cultural barriers, and learning. Strategic Management Journal, 17 (2), pp. 151-166.

Barkema, H.G., Shenkar, O., Vermeulen, F. and Bell, J.H. (1997). Working abroad, working with others: How firms learn to operate international joint ventures. Academy of Management Journal, 40 (2), pp. 426-442.

Bartlett, C.A. and Ghoshal, S. (1989). Managing Across Borders: The Transnational Solution. Boston, MA: Harvard Business School Press. 
Beaver, D. D. (2001). Reflections on scientific collaboration (and its study): past, present, and future. Scientometrics, 52 (3), pp. 365-377.

Bergstrom, C. (2007). Eigenfactor: Measuring the value and prestige of scholarly journals. College and Research Libraries News, 68, pp. 314-316.

Bergstrom, C.T., West, J.D. and Wiseman, M.A. (2008). The eigenfactor ${ }^{\mathrm{TM}}$ metrics. Journal of Neuroscience, 28, pp. 11433-11434.

Berry, H., Guillén, M.F. and Zhou, N. (2010). An institutional approach to cross-national distance. Journal of International Business Studies, 41, pp. 1460-1480.

Björkman, I., Stahl, G.K. and Vaara, E. (2007). Cultural differences and capability transfer in cross-border acquisitions: The mediating roles of capability complementarity, absorptive capacity, and social integration. Journal of International Business Studies, 38 (4), pp. 658-672.

Blomstermo, A., Sharma, D.D. and Sallis, J. (2006). Choice of foreign market entry mode in service firms. International Marketing Review, 23 (2), pp. 211-229.

Brannen, M.Y. and Salk, J.E. (2000). Partnering across borders: Negotiating organizational culture in a German-Japanese joint venture. Human Relations, 53 (4), pp. 451-487.

Brock, D.M., Shenkar, O., Shoham, A. and Siscovick, I. (2008). National culture and expatriate deployment. Journal of International Business Studies, 39 (8), pp. 1293-1309.

Brouthers, K.D. (2002). Institutional, cultural and transaction cost influences on entry mode choice and performance. Journal of International Business Studies, 33 (2), pp. 203-221.

Brouthers, K.D. and Brouthers, L.E. (2001). Explaining the national cultural distance paradox. Journal of International Business Studies, 32 (1), pp. 177-189.

Buckley, P.J. and Casson, M.C. (1976). The Future of the Multinational Enterprise. London: McMillan

Campbell, J.T., Eden, L. and Miller, S.R. (2012). Multinationals and corporate social responsibility in host countries: Does distance matter? Journal of International Business Studies, 43, pp. 84-106.

Chakrabarti, R., Gupta-Mukherjee, S. and Jayaraman, N. (2009). Mars-Venus marriages: Culture and cross-border M\&A. Journal of International Business Studies, 40, pp. 216-236.

Chandy, P.R. and Williams, T.G.E. (1994). The impact of journals and authors on international business research: A citational analysis. Journal of International Business Studies, 25 (4), pp. 715-728. 
Chang, S.J. and Rosenzweig, P.M. (2001). The choice of entry mode in sequential foreign direct investment. Strategic Management Journal, 22 (8), pp. 747-776.

Chari, M. and Chang, K. (2009). Determinants of the share of equity sought in CBAs. Journal of International Business Studies, 40, pp. 1277-1297.

Chen, G., Kirkman, B.L., Kim, K., Farh, C.I.C. and Tangirala, S. (2010). When does crosscultural motivation enhance expatriate effectiveness? A multilevel investigation of the moderating roles of subsidiary support and cultural distance. Academy of Management Journal, 53 (5), pp. 1110-1130.

Cohen, W.M. and Levinthal, D.A. (1990). Absorptive capacity: A new perspective on learning and innovation. Administrative Science Quarterly, 35 (1), pp. 128-152.

Colledge, L., Moya-Anegón, F., Guerrero-Bote, V., López-Illescas, C., El Asiati, M. and Moed, H.F. (2010). SJR and SNIP: Two new journal metrics. Serials, 23 (3), pp. 215-220.

Crossland, C. and Hambrick, D.C. (2011). Differences in managerial discretion across countries: How nation-level institutions affect the degree to which CEOs matter. Strategic Management Journal, 32, pp. 797-819.

Dagnino, G., Levanti, G., Minà, A. and Picone, P. (2015). Interorganizational network and innovation: A bibliometric study and proposed research agenda. Journal of Business and Industrial Marketing, 30 (3/4), pp. 354-377.

De Solla, P.D. (1963). Little Science, Big science. New York: Columbia University Press.

Delios, A. and Henisz, W.J. (2003a). Policy uncertainty and the sequence of entry by Japanese firms, 1980-1998. Journal of International Business Studies, 34 (3), pp. 227-241.

Delios, A. and Henisz, W.J. (2003b). Political hazards, experience, and sequential entry strategies: The international expansion of Japanese firms, 1980-1998. Strategic Management Journal, 24 (11), pp. 1153-1164.

Demirbag, M., Glaister, K.W. and Tatoglu, E. (2007). Institutional and transaction cost influences on MNEs' ownership strategies of their affiliates: Evidence from an emerging market. Journal of World Business, 42, pp. 418-434.

Demirbag, M., Tatoglu, E. and Glaister, K.W. (2008). Factors affecting perceptions of the choice between acquisition and greenfield entry: The case of western FDI in an emerging market. Management International Review, 48 (1), pp. 5-38.

Diodato V. 1994. Dictionary of Bibliometrics. Binghamton, NY: Haworth Press. 
Dow, D. and Ferencikova, S. (2010). More than just national cultural distance: Testing new distance scales on FDI in Slovakia. International Business Review, 19, pp. 46-58.

Dow, D. and Larimo, J. (2009). Challenging the conceptualization and measurement of distance and international experience in entry mode choice research. Journal of International Marketing, 17 (2), pp. 74-98.

Drogendijk, R. and Slangen, A. (2006). Hofstede, Schwartz, or managerial perceptions? The effects of different cultural distance measures on establishment mode choices by multinational enterprises. International Business Review, 15, pp. 361-380.

Ellis, P.D. (2008). Does psychic distance moderate the market size-entry sequence relationship? Journal of International Business Studies, 39 (3), pp. 351-369.

Erramilli, M.K. and Rao, C.P. (1993). Service firms' international entry-mode choice: A modified transaction-cost analysis approach. Journal of Marketing, 57 (3), pp. 19-38.

Evans, J. and Mavondo, F.T. (2002). Psychic distance and organizational performance: An empirical examination of international retailing operations. Journal of International Business Studies, 33 (3), pp. 515-532.

Evans, J., Treadgold, A. and Mavondo, F.T. (2000). Psychic distance and the performance of international retailers. A suggested theoretical framework. International Marketing Review, 17 (4/5), pp. 373-391.

Ferner, A., Quintanilla, J. and Var, M.Z. (2001). Country-of-origin effects, host-country effects, and the management of HR in multinationals: German companies in Britain and Spain. Journal of World Business, 36 (2), pp. 107-127.

Ferreira, M.P, Santos, J., Almeida, M. and Reis, N. (2014a). Mergers and acquisitions research: A bibliometric study of top strategy and international business journals, 1980-2010. Journal of Business Research, 67 (12), pp. 2550-2558.

Ferreira, M.P., Li, D., Reis, N. and Serra, F. (2014b). Culture in international business research: A bibliometric study in four top IB journals. Management Research, 12, pp. 68-91.

Ferreira, M.P., Pinto, C. and Serra, F. (2014c). The transaction costs theory in international business research: a bibliometric study over three decades. Scientometrics, 98, pp. 1899-1922.

Filatotchev, I., Strange, R., Piesse, J. and Lien, Y.C. (2007). FDI by firms from newly industrialised economies in emerging markets: Corporate governance, entry mode and location. Journal of International Business Studies, 38 (4), pp. 556-572. 
Floyd, S.W., Schroeder, D.D. and Finn, D.M. (1994). Only if I'm first author. Conflict over credit in management scholarship. Academy of Management Journal, 37 (3), pp. 734-747.

Garfield, E. (1979). Citation Indexing: Its Theory and Applications in Science, Technology and Humanities. New York: Wiley.

Gatignon, H. and Anderson, E. (1988). The multinational corporation's degree of control over foreign subsidiaries: An empirical test of a transaction cost explanation. Journal of Law, Economics and Organization, 4 (2), pp. 305-336.

Glänzel, W. (2001). National characteristics in international scientific co-authorship. Scientometrics, 51 (1), pp. 69-115.

Glänzel, W. (2009). The multi-dimensionality of journal impact. Scientometrics, 78 (2), pp. 355374.

Glänzel, W. and Moed, H.F. (2002). Journal impact measures in bibliometric research. Scientometrics, 53 (2), pp.171-193.

Glänzel, W. and Schoepflin, U. (1999). A bibliometric study of reference literature in the sciences and social sciences. Information Processing and Management, 35, pp. 31-44.

Glänzel, W. and Schubert, A. (1985). Price distribution. An exact formulation of price's square root law. Scientometrics, 7 (3), pp 211-219.

Gomez-Mejia, L.R. and Balkin, D.B. (1992). Determinants of faculty pay: An agency theory perspective. Academy of Management Journal, 35 (5), pp. 921-955.

Gong, Y. (2003) Subsidiary staffing in multinational enterprises: Agency, resources, and performance. Academy of Management Journal, 46 (6), pp. 728-739.

González-Pereira, B., Guerrero-Bote, V. and Moya-Anegón, F. (2010). A new approach to the metric of journals' scientific prestige: The SJR indicator. Journal of Informetrics, 4, pp. 379391.

Griffith, D.A., Cavusgil, S.T. and Xu, S. (2008). Emerging themes in international business research. Journal of International Business Studies, 39 (7), pp. 1220- 1235

Griffith, D.A., Hu, M.Y. and Ryans Jr., J.K. (2000). Process standardization across intra- and inter-cultural relationships. Journal of International Business Studies, 31 (2), pp. 303-324.

Guerrero-Bote, V. and Moya-Anegón, F. (2012). A further step forward in measuring journals' scientific prestige: The SJR2 indicator. Journal of Informetrics, 6, pp. 674-688. 
Hair, J., Anderson, R. and Tatham, R. (1979). Multivariate Data Analysis: With Readings. Tulsa: PPC Books.

Hall, E.T. (1976). Beyond culture. New York: Doubleday.

Hartmann, E., Feisel, E. and Schober, H. (2010). Talent management of western MNCs in China:

Balancing global integration and local responsiveness. Journal of World Business, 45, pp. 169178.

Harzing, A.W. (2002). Are our referencing errors undermining our scholarship and credibility?

The case of expatriate failure rates. Journal of Organizational Behavior, 23, pp. 127-148.

Harzing, A.W. (2003). The role of culture in entry mode studies: From neglect to myopia?. In

Cheng; J. and Hitt, M.A. (eds), Managing Multinationals in a Knowledge Economy: Economics, Culture (Advances in International Management, Volume 15). Emerald Group Publishing Limited, pp.75 - 127.

Hawkins, D.T. (1977). Unconventional uses of on-line information retrieval systems: on-line bibliometric studies. Journal of the American Society for Information Science, 28, pp. 13-18.

Hennart, J.F. (1988). A transaction costs theory of equity joint ventures. Strategic Management Journal, 9 (4), pp. 361-374.

Hennart, J.F. and Larimo, J. (1998). The impact of culture on the strategy of multinational enterprises: Does national origin affect ownership decisions?. Journal of International Business Studies, 29 (3), pp. 515-538.

Hennart, J.F. and Reddy, S. (1997). The choice between mergers/acquisitions and joint ventures: The case of Japanese investors in the United States. Strategic Management Journal, 18 (1), pp. $1-12$.

Hennart, J.F. and Zeng, M. (2002). Cross-cultural differences and joint venture longevity. Journal of International Business Studies, 33 (4), pp. 699-716.

Hofstede, G. (1980). Culture's Consequences: International Differences in Work-Related Values. Beverly Hills: Sage Publications.

Hofstede, G. (1991). Cultures and Organizations: Software of the Mind. London: McGraw-Hill.

Hood, W.W. and Wilson, C.S. (2001). The literature on bibliometrics, scientometrics, and informetrics. Scientometrics, 52 (2), pp. 291-314.

House, R., Hanges, P., Javidan, M., Dorfman, P. and Gupta, V. (2004). Culture, Leadership and Organizations: The GLOBE Study of 62 Societies. Thousand Oaks, CA: Sage. 
Hutzschenreuter, T. and Voll, J.C. (2008). Performance effects of "added cultural distance" in the path of international expansion: The case of German multinational enterprises. Journal of International Business Studies, 39, pp. 53-70.

Inkpen, A.C. and Beamish, P.W. (1994). An analysis of twenty-five years of research in the journal. Journal of International Business Studies, 25, pp. 703-713.

Jean, R.J.B., Sinkovics, R.R. and Kim, D. (2010). Drivers and performance outcomes of relationship learning for suppliers in cross-Border customer-supplier relationships: The role of communication culture. Journal of International Marketing, 18 (1), pp. 63-85.

Johanson, J. and Vahlne, J.E. (1977). The internationalization process of the firm - A model of knowledge development and increasing foreign market commitments. Journal of International Business Studies, 8 (1), pp. 23-32.

Johanson, J. and Vahlne, J.E. (2009). The Uppsala internationalization process model revisited: From liability of foreignness to liability of outsidership. Journal of International Business Studies, 40, pp. 1411-1431.

Johanson, J. and Wiedersheim-Paul, F. (1975). The internationalization of the firm - Four Swedish cases. Journal of Management Studies, 12 (3), pp. 305-323.

Johnson, J.P., Lenartowicz, T. and Apud, S. (2006). Cross-cultural competence in international business: Toward a definition and a model. Journal of International Business Studies, 37 (4), pp. 525-543.

Katsikeas, C.S., Skarmeas, D. and Bello, D.C. (2009). Developing successful trust-based international Exchange relationships. Journal of International Business Studies, 40, pp. 132155.

Kim, W. C. and Hwang, P. (1992). Global strategy and multinationals' entry mode choice. Journal of International Business Studies, 23 (1), pp. 29-53.

Knight, G.A., Hult, G.T.M. and Bashaw, R.E. (2000). Research productivity in the Journal of Business Research: 1985-1999. Journal of Business Research, 49 (3), 313-314.

Kochen, M. (1987). How well do we acknowledge intellectual debts?. Journal of Documentation, $43(1)$, pp.54 - 64 .

Kogut, B. (1988). Joint ventures: Theoretical and empirical perspectives. Strategic Management Journal, 9 (4), 319-332. 
Kogut, B. and Singh, H. (1988). The effect of national culture on the choice of entry mode. Journal of International Business Studies, 19 (3), pp. 411-432.

Kostova, T. (1999). Transnational transfer of strategic organizational practices: A contextual perspective. Academy of Management Review, 24 (2), pp. 308-324.

Kumar, V. and Kundu, S. (2004). Ranking the international business schools: faculty publication as the measure. Management International Review, 44, pp. 213-228.

Lahiri, S. and Kumar, V. (2012). Ranking international business institutions and faculty members using research publication as the measure update and extension of prior research. Management International Review, 52, pp. 317-340.

Larsson, R. and Lubatkin, M. (2001). Achieving acculturation in mergers and acquisitions: An international case study. Human Relations, 54 (12), pp. 1573-1607.

Li, J. and Tsui, A.S. (2002). A citation analysis of management and organization research in the Chinese context: 1984-1999. Asia Pacific Journal of Management, 19, pp. 87-107.

Li, J., Lam, K. and Qian, G. (2001). Does culture affect behavior and performance of Firms? The case of joint ventures in China. Journal of International Business Studies, 32 (1), pp. 115-131.

López-Duarte, C., Vidal-Suárez, M.M. and González-Díaz, B. (2015). International business and national culture: A literature review and research agenda. International Journal of Management Reviews, DOI: 10.1111/ijmr.12070.

Luo, Y. (2001). Antecedents and consequences of personal attachment in cross-cultural cooperative ventures. Administrative Science Quarterly, 46 (2), pp. 177-201.

Luo, Y. (2002). Building trust in cross-cultural collaborations: Toward a contingency perspective. Journal of Management, 28 (5), pp. 669-694.

Luo, Y. (2005). How important are shared perceptions of procedural justice in cooperative alliances? Academy of Management Journal, 48 (4), pp. 695-709.

Mäkelä, K., Björkman, I. and Ehrnrooth, M. (2010). How do MNCs establish their talent pools? Influences on individuals' likelihood of being labeled as talent. Journal of World Business, 45, pp. 134-142.

Makino, S. and Neupert, K.E. (2000). National culture, transaction costs, and the choice between joint venture and wholly owned subsidiary. Journal of International Business Studies, 31 (4), pp. 705-713. 
Manev, I.M. and Stevenson, W.B. (2001). Nationality, cultural distance, and expatriate status: Effects on the managerial network in a multinational enterprise. Journal of International Business Studies, 32 (2), pp. 285-303.

Mizruchi, M. and Fein, L. (1999). The social construction of organizational knowledge: A study of the uses of coercive, mimetic, and normative isomorphism. Administrative Science Quarterly, 44, pp. 653-683.

Moed, H.F. (2010). Measuring contextual citation impact of scientific journals. Journal of Informetrics, 4, pp. 265-277.

Moed, H.F., Colledge, L., Reedijk, J., Moya-Anegon, F., Guerrero-Bote, V., Plume, A. and Amin, M. (2012). Citation-based metrics are appropriate tools in journal assessment provided that they are accurate and used in an informed way. Scientometrics, 92, pp. 367-376.

Moed, H.F., de Bruin, R.E. and van Leeuwen, Th.N. (1995). New bibliometric tools for the assessment of national research performance: Database description, overview of indicators and first applications. Scientometrics, 33, pp. 381-422.

Morgan, R.M. and Hunt, S.D. (1994). The commitment-trust theory of relationship marketing. Journal of Marketing, 58 (3), pp. 20-38.

Morosini, P., Shane, S. and Singh, H. (1998). National cultural distance and cross-border acquisition performance. Journal of International Business Studies, 29 (1), pp. 137-158.

Morrison, A.J. and Inkpen, A.C. (1991). An analysis of significant contributions to the international business literature. Journal of International Business Studies, 22, pp. 143-154.

Morschett, D., Schramm-Klein, H. and Swoboda, B. (2010). Decades of research on market entry modes: What do we really know about external antecedents of entry mode choice? Journal of International Management, 16, pp. 60-77.

O'Grady, S. and Lane, H.W. (1996). The psychic distance paradox. Journal of International Business Studies, 27 (2), pp. 309-333.

Park, S.H. and Ungson, G.R. (1997). The effect of national culture, organizational complementarity, and economic motivation on joint venture dissolution. Academy of Management Journal, 40 (2), pp. 279-307.

Parkhe, A. (1991). Interfirm diversity, organizational learning, and longevity in global strategic alliances. Journal of International Business Studies, 22 (4), pp. 579-601. 
Paterson, S.L. and Brock, D.M. (2002). The development of subsidiary-management research: Review and theoretical analysis. International Business Review, 11, pp. 139-163.

Peng M.W. and Zhou, J.Q. (2006). Most cited articles and authors in global strategy research. Journal of International Management, 12, pp. 490-508.

Pinto, C., Serra, F. and Ferreira, M.P. (2014). A bibliometric study on culture research in international business. Brazilian Administration Review, 11 (3), pp. 340-363.

Pisani, N. (2011). International management research: Investigating its recent diffusion in top management journal. Journal of Management, 35 (2), pp. 199-218.

Podsakoff, P.M., MacKenzie, S.B., Bachrach, D.G. and Podsakoff, N.P. (2005). The influence of management journals in the 1980s and 1990s. Strategic Management Journal, 26, pp. 473488.

Pothukuchi, V., Damanpour, F., Choi, J., Chen, C.C. and Park, S.H. (2002). National and organizational culture differences and international joint venture performance. Journal of International Business Studies, 33 (2), pp. 243-265.

Pritchard, A. (1969). Statistical bibliography or bibliometrics? Journal of Documentation, 24, pp. 348-349.

Quer, D., Claver, E. and Rienda, L. (2007). Business and management in China: A review of empirical research in leading international journals. Asia Pacific Journal of Management, 24, pp. 359-384.

Ramos-Rodríguez, A.R. and Ruíz-Navarro, J. (2004). Changes in the intellectual structure of strategic management research: a bibliometric study of the Strategic Management Journal, 1980-2000. Strategic Management Journal, 25, pp. 981-1004.

Reus, T.H. and Lamont, B.T. (2009). The double-edged sword of cultural distance in international acquisitions. Journal of International Business Studies, 40, pp. 1298-1316.

Reus, T.H. and Rottig, D. (2009). Meta-analyses of international joint venture performance determinants. Evidence for theory, methodological artifacts and the unique context of China. Management International Review, 49, pp. 607-640.

Ronen, S. and Shenkar, O. (1985). Clustering countries on attitudinal dimensions: A review and synthesis. Academy of Management Review, 10 (3), pp. 435-454. 
Sakarya, S., Eckman, M. and Hyllegard, K.H. (2007). Market selection for international expansion: Assessing opportunities in emerging markets. International Marketing Review, 24 (2), pp. 208-238.

Sarala, R.M. and Vaara, E. (2010). Cultural differences, convergence, and crossvergence as explanations of knowledge transfer in international acquisitions. Journal of International Business Studies, 41, pp. 1365-1390.

Schlegelmilch, B.B. and Chini, T.C. (2003). Knowledge transfer between marketing functions in multinational companies: a conceptual model. International Business Review, 12, pp. 215-232.

Schwens, C., Eiche, J. and Kabst, R. (2011). The moderating impact of informal institutional distance and formal institutional risk on SME entry mode choice. Journal of Marketing Studies, 48 (2), pp. 330-351.

Shafique, M. (2013). Thinking inside the box? Intellectual structure of the knowledge base of innovation research (1988-2008). Strategic Management Journal, 34 (1), pp. 62-93.

Shenkar, O. (2001). Cultural distance revisited: Towards a more rigorous conceptualization and measurement of cultural differences. Journal of International Business Studies, 32 (3), pp. $519-535$.

Shenkar, O., Luo, Y. and Yeheskel, O. (2008). From "distance" to "friction": Substituting metaphors and redirecting intercultural research. Academy of Management Review, 33 (4), pp. 905-923.

Shimizu, K., Hitt, M.A., Vaidyanath, D. and Pisano, V. (2004). Theoretical foundations of crossborder mergers and acquisitions: A review of current research and recommendations for the future. Journal of International Management, 10, pp. 307-353.

Sicilia, M.A., Sánchez-Alonso, S. and García-Barriocanal, E. (2011). Comparing impact factors from two different citation databases: The case of computer science. Journal of Informetrics, 5, pp. 698-704.

Sirmon, D.G. and Lane, P.J. (2004). A model of cultural differences and international alliance performance. Journal of International Business Studies, 35 (4), pp. 306-319.

Skarmeas, D., Katsikeas, C.S. and Schlegelmilch, B.B. (2002). Drivers of commitment and its impact on performance in cross-cultural buyer-seller relationships: The importer's perspective. Journal of International Business Studies, 33 (4), pp. 757-783. 
Slangen, A.H.L. (2006). National cultural distance and initial foreign acquisition performance: The moderating effect of integration. Journal of World Business, 41, pp. 161-170.

Slangen, A.H.L. and Van Tulder, R.J.M. (2009). Cultural distance, political risk, or governance quality? Towards a more accurate conceptualization and measurement of external uncertainty in foreign entry mode research. International Business Review, 18, pp. 276-291.

Smith, L. (1981). Citation analysis. Library Trends, 30 (1), pp. 83-106.

Sousa, C.M.P., Ruzo, E. and Losada, F. (2010). The key role of managers' values in exporting: Influence on customer responsiveness and export performance. Journal of International Marketing, 18 (2), pp. 1-19.

Stahl, G.K. and Caligiuri, P. (2005). The effectiveness of expatriate coping strategies: The moderating role of cultural distance, position level, and time on the international assignment. Journal of Applied Psychology, 90 (4), pp. 603-615.

Stahl, G.K., Maznevski, M.L., Voigt, A. and Jonsen, K. (2010). Unraveling the effects of cultural diversity in teams: A meta-analysis of research on multicultural work groups. Journal of International Business Studies, 41, pp. 690-709.

Stahl, M.J., Leap, T.L. and Wei, Z.Z. (1988). Publication in leading management journals as a measure of institutional research productivity. Academy of Management Journal, 31 (3), pp. 707-720.

Starbuck, W. (1994). Journals ranked by citations per article. Working Paper. NYU Stern School of Business.

Tahai, A. and Meyer, M.J. (1999). A revealed preference study of management journals' direct influences. Strategic Management Journal, 20, pp. 279-296.

Takeuchi, R., Tesluk, P.E., Yun, S. and Lepak, D.P. (2005). An integrative view of international experience. Academy of Management Journal, 48 (1), pp. 85-100.

Tihanyi, L., Griffith, D.A. and Russell, C.J. (2005). The effect of cultural distance on entry mode choice, international diversification, and MNE performance: A meta-analysis. Journal of International Business Studies, 36 (3), pp. 270-283.

Tong, T.W. and Reuer, J.J. (2007). Real options in multinational corporations: Organizational challenges and risk implications. Journal of International Business Studies, 38 (2), pp. 215230. 
Treviño, L.J., Mixon Jr.F.G., Funk, C.A. and Inkpen, A.C. (2010). A perspective on the state of the field: International business publications in the elite journals as a measure of institutional and faculty productivity. International Business Review, 19, pp. 378-387.

Trieschmann, J.S., Dennis, A.R., Northcraft, G.B. and Niemi, A.W.Jr. (2000). Serving multiple constituencies in business schools: M.B.A. program versus research performance. Academy of Management Journal, 43 (6), pp. 1130-1141.

Trompenaars, F. and Hampden-Turner, C. (1993). Riding the Waves of Culture: Understanding Cultural Diversity in Business. London: Nicholas Brealey Publishing.

Uhlenbruck, K. (2004). Developing acquired foreign subsidiaries: The experience of MNEs in transition economies. Journal of International Business Studies, 35 (2), pp. 109-123.

Vaara, E., Sarala, R., Stahl, G.K. and Björkman, I. (2012). The impact of organizational and national cultural differences on social conflict and knowledge transfer in international acquisitions. Journal of Management Studies, 49 (1), pp. 1-27.

Vaara, E., Tienari, J. and Santti, R. (2003). The international match: Metaphors as vehicles of social identity-building in cross-border mergers. Human Relations, 56 (4), pp. 419-451.

Van Vianen, A.E., De Pater, I.E., Kristof-Brown, A.L. and Johnson, E.C. (2004). Fitting in: Surface- and deep-level cultural differences and expatriates' adjustement. Academy of Management Journal, 47 (5), pp. 697-709.

Waldman, D.A., Luque, M.S., Washburn, N., House, R.J., Adetoun, B., Barrasa, A., Bobina, M., Bodur, M., Chen, Y.J., Debbarma, S., Dorfman, P., Dzuvichu, R.R., Evcimen, I., Fu, P., Grachev, M., Gonzalez Duarte, R., Gupta, V., Hartog, D.N., Hoogh, A.H.B., Howell, J., Jone, K.Y., Kabasakal, H., Konrad, E., Koopman, P. L., Lang, R., Lin, C.C., Liu, J., Martinez, B., Munley, A.E., Papalexandris, N., Peng, T., Prieto, L., Quigley, N., Rajasekar, J., Gil Rodriguez, F., Steyrer, J., Tanure, B., Thierry, H., Thomas, Fr.V.M., Berg, P.T. and Wilderom, C.P.M. (2006). Cultural and leadership predictors of corporate social responsibility values of top management: A GLOBE study of 15 countries. Journal of International Business Studies, 37 (6), pp. 823-837.

Waltman, L., van Eck, N.J., van Leeuwen, T.N. and Visser, M.S. (2013). Some modifications to the SNIP journal impact indicator. Journal of Informetrics, 7, pp. 272-285.

Weber, Y., Shenkar, O. and Raveh, A. (1996). National and corporate cultural fit in mergers/acquisitions: An exploratory study. Management Science, 42 (8), pp. 1215-1227. 
Werner, S. (2002). Recent development in international management research: A review of 20 top management journals. Journal of Management, 28 (3), pp. 277-305.

White, D. and McCain, K. (1998). Visualizing a discipline: An author co-citation analysis of information science, 1972-1995. Journal of the American Society for Information Documentation, 43 (1), pp. 54-64.

Williamson, O. (1985). The Economic Institutions of Capitalism. New York: Free Press.

Wu, F., Sinkovics, R.R., Cavusgil, S.T. and Roath, A.S. (2007). Overcoming export manufacturers' dilemma in international expansion. Journal of International Business Studies, $38(2)$, pp. 283-302.

$\mathrm{Xu}$, S., Yalcinkaya, G. and Seggie, S.H. (2008). Prolific authors and institutions in leading international business journals. Asia Pacific Journal Management, 25, pp. 189-207.

Zaheer, S. (1995). Overcoming the liability of foreignness. Academy of Management Journal, 38 (2), pp. 341-363.

Zaheer, S. and Zaheer, A. (2006). Trust across borders. Journal of International Business Studies, 37 (1), pp. 21-29.

Zhang, C., Cavusgil, S.T. and Roath, A.S. (2003). Manufacturer governance of foreign distributor relationships: Do relational norms enhance competitiveness in the export market? Journal of International Business Studies, 34 (6), pp. 550-566.

Zupic, I. and Čater, T. (2015). Bibliometric methods in management and organization. Organizational Research Methods, 18 (3), pp. 429-472. 
